\section{EL COLEGIO DE HUÉRFANOS DE FERROVIARIOS DE \\ TORREMOLINOS. UN EJEMPLO DE INNOVACIÓN DOCENTE}

DESDE EL PROYECTO ARQUITECTÓNICO

THE TORREMOLINOS SCHOOL FOR ORPHANS OF RAILWAY WORKERS. AN EXAMPLE OF EDUCATIONAL INNOVATION AS ARCHITECTURAL PROJECT

Mar Loren-Méndez; Daniel Pinzón-Ayala; Ana Belén Quesada-Arce
RESUMEN El antiguo Colegio de Huerfanos de Ferroviarios de Torremolinos (Malaga) (1933/1935) es una obra ampliamente

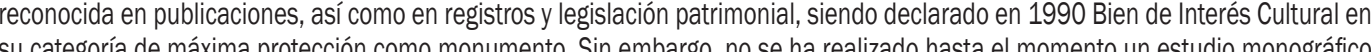

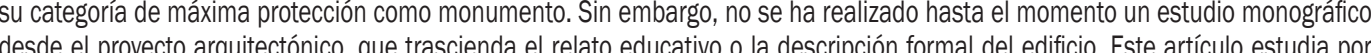
政 autor de todos los proyectos de la red de Colegios de Huérfanos de Ferroviarios (CHF), presentando el CHF de Torremolinos como la propuesta de referencia. Comenzando con una presentiacion de la asociación CHF y del autor, el colegio de Torremolinos se analiza desde un a triple entrada: la búsqueda desde el proyecto en el marco del debate tipológico, la formalización de las corrientes de innoPALABRAS CLAVES Colegio de Huérfanos de Ferroviarios; Francisco Alonso Martos; arquitectura escolar; arguitectura moderna; Arquitectura Contemporánea de Andalucía; Málaga.

SUMMARY The former Torremolinos School for Orphans of Railway Workers in the province of Malaga in Spain (1933/1935) is a work of architiecture that has been widely referernced in publications, registers and heritage legisilation, declared an Asset of Cutural sessment of the proiect has been carried out moving beyond ed cational or formal descriptions of the buildins The orticle presents this type of hybrid socio-educational architecture in its historical context for the first time, focusing on the work of Francisco Alonso Martos, the architect behind all the projects that comprise a network of Schools for Orphans of Railway Workers (SORs). A general overview of the SOR network and its architect is followed by a specific study of the Torremolinos SOR, considering three main aspects: its place in the typological debate, the for foll KEY WORDS School for Orphans of Railway Workers; Francisco Alonso Martos; educational architecture; modern architecture; contemporary architecture in Andalusia; Malaga.

Persona de contacto/Corresponding author: marloren@us.es. Escuela Técnica Superior de Arquitectura. Universidad de Sevilla. España.

\section{DIMENSIÓN EDUCATIVADE LA} VOCACIÓN SOCIAL FERROVIARIA

El Colegio de Huérfanos de Ferroviarios y su arquitecto de cabecera: Francisco Alonso Martos

E n 1922 se funda la organización benéfica Colegio de Huérfanos Ferroviarios (CHF), como filia de la Asociación de Empleados y Obreros de os Ferrocarriles de España (AGEOFE), creada en 1888 Ya en 1912 se documenta un movimiento ferroviario proponiendo la creación de estos equipamientos para huérfanos, denominándose en principio como "asilos" La dimensión educativa como parámetro fundamental de su misión social queda definida con la Real Orden de 12 de mayo de 1926, identificándose con un perfil mixto asistencial y docente. Se aproxima así a las asociaciones que hoy denominamos de acción social, predominando la vocación educativa.

En 1935 se especificaba que para cumplir su finaldad, la asociación CHE "construirá y sostendra Colegios

. SANTOS, José. El Assilo de Huérfanos Ferroviarios. Una idea plausible. En: AAdelantel... Revista Semanal Ilustrada de Ferrocarriles, nº 61,1912, pp. 5-6. 2. Estatututo del 1 de jullio de 1935, art. 20. Estatutuos del CHF - Antecedentes [en línea]. Asociación CHF [consulta: 21-01-2017]. Disponible en: http:///WwW. sff-cogt.com/CHF/ESTATUTOS/Antecedentes.pdf., p. 3.

3. loíd, p. 4.

4. Arquitecto de la AGEOFE, del Internado de Pensionistas Ferroviarios, de la Asociación Ferroviaria Médico-Farmacéutica, de la cooperativa el Hogar Ferrovi rio, la cooperativiv Ferroviariay C Ciudadedes eerroviarias. Sobre sus obras: Cfr. La Asociación general de empleados y obreros de los Ferrocarriles de España y y otros establecimientos para la educación e instrucción ge los humanos". Apu pla asia la construcción de coleéstos cobrá́n tal importancia en símismos que terminarian por confundirse con el fin"3 Es significativo el nombre elegido para la asociación enfatizando desde su origen el papel fundamental que el espacio docente tiene en el uturo de los huérfanos.

Esta investigación desvela que Francisco Alonso Maros (1886, Granada /1961, Madrid t. 1913) fue el arquitecto contratado para llevar a cabo dichas construcciones. Junto con los colegios de huérfanos de ferroviarios, Alonso Martos realiza una red de colegios, algunos de los cuales incluyen en su programa sedes sociales. El arquitecto proyecta asi mismo ciudades-jardín ferroviarias -coniuntos autosuficientes donde siempre se incluía un proyecto de escuela- aunque no llegaron a construirse. Se convirtió en el técnico de una producción significativa en la definición de sus distintos 政 organizaciones ferroviarias de vocación social4 teniendo 
1. Localización, datos cronológicos y esquema en
planta de los $C H F$.

2. Portada del Boletin Oficial del Colegio de Huérfa

nos de Ferroviarios, $n^{\circ}$ extraordinario, 1930. espacios docentes. Además, como arquitecto de la Dirección General de Prisiones, abordó el campo de la rehabililación social a través de la formación en colonias agrícolas penitenciarias recogidas en la Ley relativa a vagos y maleantes de 1933, realizando los proyectos de la lsla de Ons (Pontevedra), de Oña (Burgos) y una propuesta similar para Guinea Ecuatorial. También fue arquitecto de numerosas instituciones públicas y privadas: del Catastro de la Riqueza El Pardo (Madrid), de la Diócessis de Madrid y de diferentes El Pardo (Madrid), de la Diócesis de

La organización CHF promovió un total de diez grandes complejos entre 1926 y 1958: Madrid, Torremolinos (Malaga), Alicante, Palencia, León, Avila, Vilagarcia de Arousa (Pontevedra), Dos Hemanas (Sevila), Hondamrimeros (Gipara) y Vacarisses (Barcelona). Solo los dos

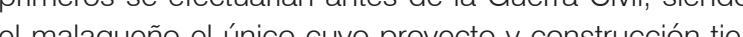

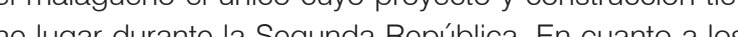
estantes, el de Vilagarcía de Arousa finat en funcionamiento $\mathrm{COmO}^{\mathrm{C} \mathrm{CH}^{6}}$ y los de Hondatio carisses $\mathrm{Dos}$ Ho como CH y y los de Honclanbia, Vaultimo se concibí como Colio Maternat, contemplando un programa algo difente (figura 1). En la dictadura a la del arquitecto se desarrolla entre fuetes citicas, con una visión negativa de sus proyectos. Esto provoca su renuncia on 1951 como arquiecto conservador de colegios existentes Madrid y Torremolinos- y en 1953 como aquitecto y director de obras de los que se encontraban en fase de ejecuc

INNOVACIÓN PEDAGÓGICA Y ARQUITECTURA

La propuesta educativa de los CHF formalizaba "los va-

ores de progreso y modernidad ${ }^{17}$ que caracterizaban a

las asociaciones vinculadas al ferrocarril (figura 2). Esta visión encuentra su acomodo en las reformas del sistema educativo de las primeras décadas del siglo XX, con un mpulso relevante en la década de los años veinte, con e objetivo de que se "aleje el clasismo de la enseñanza, supripa el monopolio clencal e incorpore las nuevas corrientes pedagogicas a una realidad escolar anquilosada"i. Pero los compromisos polficicos adquiridos por la Dictadura de Prón de Rivera desembocaron en una instrumentación ideológica de la educación que afectaría puntualmente a proyecto educativo de los CHF. en un principio se muespran religiosas". Aunque hinalmente apostanon por la educación La semuna Reaplla en el CHF de Madid.

La Segunda Republica será el régínen politico más propicio para el proyecto educalvo del CH. Las prioridades de inovación pedagogica quedan palentes con la

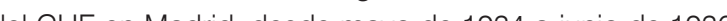
cento

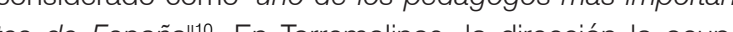
Jacinto Ruiz Santiago, con igual traycloria en innovaco codáciz Sa ditirá tras el Los planes de estudios se componen, desde Los pla segunda Enseranza, incluyndo esta úl hasta el gado de bachiler 2 n 1029 inclye el buchille elemental y el civechiller que se contemplan se establecen tanto para niños comb para niñs, con leves variociones entre los dos, acorde s "que atribuian una mayor debilidad al organismo femenino"'t

La realidad de posguerra evidenciará una falla intelecfual insalvable entre Alonso Martos y bos nuevas premisas del Régimen, a pesar de su patente compromiso con los ferroviarios Se impone una educación religiosa obligatoria como así se corrobora con la llegada de los religiosos a

5. Cfr. ALONSO MARTOS, Francisco. La Construcción de los Colegios de Huérfanos de Ferroviarios (en leǵtíma defensa). Madrid: 1951, p. 18. 6. RENFE Io adquiríí para Escuela-Internado de Aprendices. Cfr. LOZANO AGúNDEZ, Jesús. Evolución Históricico-Educativa Del Colegio de Huérfanos de Ferroviarios (CHF). Una Propuesta Socio Laboral (1922-1996). Directora: María Rosario Limón Mendizábal. Tesis Doctoral. Universidad Complutense de Madrid, Departamento de Teoría e Historia de la Educación, 2016, p. 222.

7. SÁNCHEZ COLLANTES, Sergio. Ferrocarril eideología en España a finales el XXX. Contribución a su estudio. En: I Congreso Historia Ferroviaria. Málaga: 2006. 8. LÓPEZ MARTTí, Ramón. El magisterio primario en la dictadura de Primo de Rivera. Notas para su estudio. En: Historia de la educación: revista interuniver

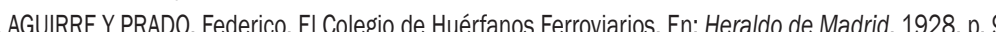

\begin{tabular}{|c|c|c|c|c|c|}
\hline Colegio & Proyecto & Primera Piedra & Inauguración & Cierre & Esquema Planta \\
\hline Madrid & 1926 & 3011/1/1926 & $0705 / 1930$ & & and \\
\hline Torremolinos & 1933 & $2101 / 1 / 1934$ & $1209 / 1935$ & $3006 / 1973$ & 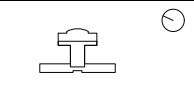 \\
\hline Alicante & 1943 & $033041 / 1945$ & $1201 / 1954$ & $3006 / 1990$ & ${ }_{\square}{ }^{\circ}$ \\
\hline Avila & 1945 & $26004 / 1946$ & $2009 / 1954$ & 300661978 & $\stackrel{0}{2}$ \\
\hline Palencia & 1943 & $1305 / 1945$ & $1505 / 1953$ & $3006 / 1982$ & 罚 \\
\hline $\begin{array}{l}\text { Vilagarcia de } \\
\text { Arousa }\end{array}$ & 1945 & $2405 / 1945$ & $26004 / 1968$ & 1984 & $\square_{\square} 0$ \\
\hline León & 1943 & 250661945 & $06 / 11 / 1958$ & $30106 / 1984$ & $\square$ \{ \\
\hline Hondarribia & 1945 & $2509 / 1946$ & & $\begin{array}{r}1971 \text { (Se revierte al } \\
\text { Ayto.) }\end{array}$ & $\left.\square^{\square}\right]^{0}$ \\
\hline $\begin{array}{l}\text { Dos } \\
\text { Hermanas }\end{array}$ & 1944 & $210041 / 945$ & & $\begin{array}{r}188121 / 1968 \\
\text { (Expropiación forzosa) }\end{array}$ & $r_{\square}{ }^{\ominus}$ \\
\hline Vacarisses & 1943 & 150661945 & & $\begin{array}{l}1953 \text { (Se revierte a su } \\
\text { propictaria) }\end{array}$ & $\underbrace{}_{\square}$ \\
\hline
\end{tabular}

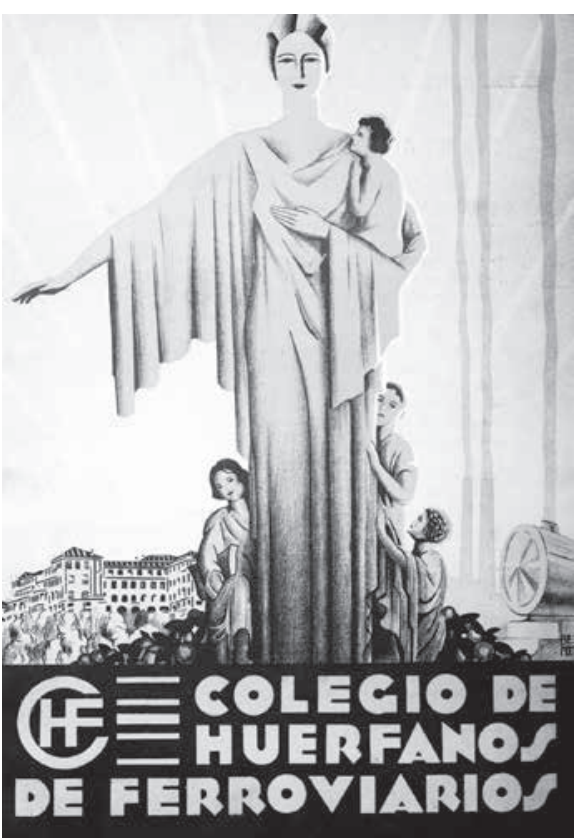

dirección de los diferentes colegios. Aun así, no habrá cambios significativos en los programas de los colegios proyectados en plena $A$ capillas, cuestión por la que se le acusará de ateo. de las nuevas concepciones pedagógicas. Éstas se canaIzaban en España, fundamentalmente, "desde la Institución Libre de Enseñanza [. . ] contribuyendo también en otras instituciones como el Museo Pedagógico Nacional"12. Estas corrientes se entrelazaron con las propuestas higienistas como método de modernización y diseño de la arquiteccomo de de arquitecescuelas al aire libre y las colonias escolares de verano ${ }^{13}$ Martos a priorizar una óptima iluminacior
Por otro lado el contexto en el que se fragua la idea de $\mathrm{CHF}$ coincide con un entorno en constante debate acerca Estas aspiraciones funcionales llevan a Alonso empleando "un moderno sistema de aireación"14; una arquitectura que mira al exterior y que establece crujias dimensionado de ventanas en relación con la orientación cada local en el sitio que debía tener, dotándole de la orientación e iluminación precisas [ ] ] Es decir que cada ventana fue donde hizo falta: cada puerta donde fue necesaria"15. Esta reflexín se distancia de las arquitecturas sanitarias en el uso terapéutico del sol: en contraste tien en cuenta los programas docente y residencial. Sopesa la orientación óptima para ambos desce el punto de vista higienista integrada con la sectorización programática y con las corrientes de innovación educativa en las que la ocupación activa-creativa al aire libre serán claves. Los CHF proponen dos elementos para asegurar este contacto directo con el entorno: por un lado, el y el uso de las estancias: "Surgió el proyecto colocando

10. LóPEZ MARTiNEZ, Damián; BERNAL MARTíNEZ, José Mariano. De la teoría pedagógica a la práctica docente: Aurelio Rodríguez Charentón y la renovvación de las ciencias escolares en el primer tercio del siglo XX. En: Homenaje al profeseror Alfonso Capitán. Murcia: Universidad de Murcia, 2005, . . 51 11. LAHOZ ABAD, Purficacaíon. Los modelos escolares de la Oficina Técnica para la construcción de escuelas. En: Historia de la Educación, 1993-1994 vol. XIIIXIII, p. 135.

12. ANON ABAAS, Rosa Marria. La Arrquitectura de las Escuelas Primarias Municipales de Sevilla hasta 1937. Sevilla: Junta de Andalucía, Universidad de Sevilla, Kora (col.)., $n^{\circ} 15,2005$, p. 37.

13. lbíd, pp. 41-47. LAHOZ ABAD, op. cit. supra, nota 11, pp. 121-148.

nos de Ferroviarios de Torremolinos. En: Blanco y Negro, 22 marzo 1936, p. 78

15. Hablendo con nuestro 
recreo cubierto, que aparece en Torremolinos como una pieza con escala suliciente para asegurar que los estudiantes puedan salir al exterior. El programa diario hacia un énfasis especial en el juego y las actividades creativas en el exterior como parte fundamental de la educacion: los jardines se dotaron de lluminación, de modo que "los niños podrán jugar de noche ${ }^{\text {mit. }}$. En Torremolinos se oriena al Este, promoviendo la vista del mar, a un bello paisaje como parte importante del desarrollo de las capacidades del niño. Por otro lado, las galerías porticadas, situadas en Torremolinos al Oeste, en contacto con las aulas, son consideradas por el arquitecto "de gran utilidad [.... y proporcionarán a los chicos un buen silio donde jugar [... con fácil comunicación con el jardín para sus recreos"'t1. La separación de estas dos zonas consiguen motivar un recorrido exterio

Recreo cubierto y galerías porticadas constituyen una constante en los distintos proyectos, aunque cada localización supondra una variante según la orientación del solar y la zona climática. Esta cualificación de los espacios exteriores formaliza el énfasis de las corrientes de innovación pedagogica en el juego del nino y el contacto con la naturaleza. En 1935 se añadiría a las unciones del director del Colegio la de preocuparse por la educación física y moral de los alumnos, atendiendo especialmente a sus juegos, recreos, salidas y distracciones como elementos utilisimos de los modernamente empleados por la Pedagogía"1"1. Un espacio libre para ser descubierto, en el que perderse y encontrar rincones propios. Las terrazas, elementos vinculados a los dormitorios, tambien serian una constante en los CHF. Con una presencia muy protagonista en el colegio de Madrid, a partir de Torremolinos se hace más puntual dentro de las aspiraciones de simplificación y eficacia dado el encarecimiento que éstas provocan. En Torremolinos se sifuan al Estecomo cubierlas, aprovechando de nuevo las vistas y de espacios de terapia.

Parámetros de salud, creatividad y oportunidad para a docencia y el juego al aire libre constituyen ahora limiaciones a la educacion promovida por el Regimen. Las aspiraciones de autosuficiencia y el control del tiempo ibre hacen que el juego en los CHF, como motor educativo, sea sustituido por labores de formación agropecuaria y un enfasis en el deporte, en el marco del adoctrinamiento de la Dictadura. Las galerias cubiertas se coniguran como extensión del programa, eliminando los grados de libertad anteriores. En el colegio de Alicante, galería porticada, esta vez abienta a Oste, hará las veces de aula y comedor de verano. En Avila, hará extensiva esta estrategia programálica al comedor, al salón de usos mulities y a una biblioteca. En este colegio es Unde unicamente aparocerán otras galerias como extensión de los dormitorios galería sanitaria-. Sin embargo, la orientación convexa -que no concava- de la fachada no busca la captación maxima solar, sino la iluminación óptima.

Junto con la sectorización docente-residencial existe además una reflexión de división por sexo. En origen los CHF plantean esquenas mixtos, reforzando la visión progresista de la educación. Para independizarlos duplican los núcleos de escaleras en el caso de Torremolinos, estableciendo plantas simetricas, ya recogidas por las mismas razones para las escuelas públicas, permitiendo que existan dormitorios para niños y niñas en ambas prientaciones. El Franquismo immone una separación por sexos, desapareciendo la sectorización vertical en os colegios. Se decide dejar los enclaves de clima más benigno para las niñas, siendo por tanto Torremolinos asignado a las comprendidas entre 8 y 12 años. La locaIzación privilegiada del colegio frente al mar se convierte rol en Sol. En epoca estival, las niñas eran trasladadas a Alicante Palencia "por aquello del ambiente externo poco colegio"20.

\section{PRADOS LÓPEZ, op. cit. supra, nota 14, p. 79}

17. El nuevo colegio. En: Boletin oficial del Colegio de huérfanos de ferroviarios, agosto $1935, n^{\circ} 94, p .6$.

8. LOZANO AGÚNDEZ, op. cit. supra, nota 6, p. 51

19. lbíd, p. 220.

EL CHF DE TORREMOLINOS EN CONTEXTO Los CHF en el marco del debate tipológico

Los colegios-internado para los huérfanos de ferroviarios comparten por tanto un carácter híbrido, que combina función benéfico-social con el compromiso principal con ducación. Se trata de equipamientos de gran escala, que trascienden el ámbito local. Descartada la opción de ocupar edificios preexistentes, la selección del solar tiene cuenta las condiciones estratégicas de soleamiento, ventiación y emplazamiento periferico, con la accesibilidad por ferrocarril dada su vinculación institucional, combinándoa con el acceso por carretera. Los edificios proyectados permitian un espacio libre mucho mayor al ocupado por el edificio, en la línea establecida tanto para las construcciones escolares como para las sanitarias ${ }^{21}$. En la época franquista las parcelas aumentarian considerablemente amaño, lo que permitía la inclusión de usos productivos agropecuarios, con lo que "se buscaba procurarse el sostenimiento de los mismos con medios propios"z?.

La reflexión tipologica de los CHF se incardina dentro de dos grupos de arquitecturas, las relacionadas con e mundo sanitario y con el docente. En cuanto a las primeas, las soluciones se debatian, básicamente, entre el uso de pabellones aislados, de ongen estadounidense, y edificio único, modelo germano-suizo ${ }^{23}$. Esta reflexión en orno a la atomización o la compactación del programa se enmarca en las teorias higienistas imperantes y en la necesidad de separar y aislar determinados usos. En el ámbito nacional se aprecia ese debate en la evolución de los hospitales antituberculosos tanto en los privados como en los públicos ${ }^{24}$. Durante la Dictadura de Primo de Rivera se tenderá hacia el edificio único en un bloque aislado²,

21. Cfr. RULLOBA QUECEDO, Cecilia. Arquitectura sanitaria: sanatorios antituberculosos. Madrid: Escuela Nacional de Sanidad, Instituto de Salud Carlos III, 22.

23. Crf. CREMNITZER, Jean-Bernard. Architecture et Santé. Le temps du sanatorium en France et en Europe. A. et J. Picard, Paris 2005, pp. 35-49. RULLOBA dolid, Departamento de Teoría de la Arquitectura y Proyectos Arquitectónicoss, 2012, pp. 115-368. . 25. El "Reglamento de los Dispensarios Antituberculosos del Real Patronato" se aprobó en 1927, en 1928 las directrices para su construcción. 26. RULLOBA QUECEDO, op. cit. supra, nota 21, p.

28. bíd, p. 80 . con ejemplos puntuales del modelo con planta en forma mo. Tras el "Concurso de Anteproyectos de Sanatorios Antituberculosos de 200, 300 y 400 camas" (1942), la solución ganadora de Ernesto Ripolles, con una planta Pe Construcciones de Lucha contra la Tuberculosis. Por otro lado, la arquitectura docente también se encarencia importante de edificaciones escolares y las exisentes presentaban gran precariedad e insalubridad. La progresiva mejora de las construcciones escolares se 政 cuelas. acción que más claramente demuestra la conin escolares de 1905, 1923 y 1934- así como la mencionada evolución de las teorlas pedagogicas e higienistas. gramas más complejos que irán más allá de las aulas, I tos to to amática era mayor. Así se manifiesta con la preocupa(a) despacios residenciales, así como por el tratamiento ( e avión o en T, indagación tipológica que continúa en la (Este debate se vincula con las reflexiones de 
3. Proyecto de Colegio de Huérfanos Ferroviarios en Malaga. Plano de emplazas

4. Colegio de Huérfanos de Ferroviarios de TorremoInos, rehabilitado como Centro Cultural Pablo Ruiz Picasso. Vista general Sureste.

proyecto en torno a la incorporación de nuevos usos tanto en las arquitecturas escolares como en las sanitarias ${ }^{29}$

Una red de nacional. Origen y definición de los $\mathrm{CHF}$ El CHF de Madrid fue el primero en proyectarse y construirse. Concebidos en red, Madrid actúa como entidad central del sistema, y el resto de colegios como sucursaes ${ }^{30}$. Mientras se decide su emplazamiento, Alonso Maros estudia referentes en España, visitando tanto colegios internados como sanatorios. El arquitecto concluye que "la inmensa mayoría no reúnen las condiciones debidas"31. Señala como excepciones el internado de Pedernales (Bizkaia); el Sanatorio Marítimo de Gorliz (Bizkaia) y el Sanatorio Marítimo en la Isla de Pedrosa (Cantabria). Todos ellos se implantan de manera estratégica y proponen un edificio único de clara axialidad, como se verá en los CHF.

En Europa, Alonso Martos destaca los internados colegios en Suiza -Ginebra, Lausanne, Vevey ${ }^{32}-$ y en Francia la Colonia Agricola de Beaubais y el Sanatorio Martimo de Hendaya. Este útimo está organizado en pabellones, hecho que demuestra que Alonso Martos esudia ambas IIneas tipologicas. No obstante, explica que existen menos insitituciones para huerfanos en el norte de Europa: la menor natalidad y una mayor previsión estatal en el caso de pérdida del progenitor-con equipamientos públicos que cubren estas demandas- apuntan a una cierta especificidad de la problemática en España.

Alonso Martos explicita la participación activa de la Asociación del CHF, considerando el proyecto la adaptación de as opiniones del Consejoy empleados: "Puedo, portanto, afirmar, que en el edificio del Colegio de Huertanos estan las ideas de todos, sin que yo haya hecho más que su adaptación"si.

El Colegio de Madrid inicia la investigación desde el proyecto de estos complejos: una reflexión tipológica del edificio único para el programa híbrido de colegio internado; las preocupaciones de orientación y zona climática como material fundamental de adaptación al lugar; la inserción de estas arquitecturas en el debate pedagógico trascendiendo la dimensión higienista; el uso extensivo del hormigón. En el marco de las preocupaciones higienistas, sólo independiza la enfermería. Aparece también la definición geométrica característica de planta en doble T que se clarifica en Torremolinos. La pieza central es ocupada en el caso de Madrid por un patio $0^{34}$, reducto de la tipologia claustral aplicada a los espacios docentes, estableciendo una tensión no resuelta entre la referencia interior del patio y la apertura del colegio al exterior mediante terrazas para los dormitorios. Siendo aún la primea aproximación a este programa y con un cierto exceso ornamental, esta propuesta construida es relevante de la vision docente y social de la AGEOFE. Definición axial y simetria caracterizan desde entonces las propuestas para los CHF, solución que se interpreta desde la doble entrada de la formación academicista del autor y las corrientes ipologicas imperantes, en busqueda de claridad programática y de estandarización del proceso constructivo.

El CHF de Torremolinos. El desarrollo de un referente El CHF de Torremolinos es la primera sucursal de la red. Estos equipamientos eran ambicionados por los distintos municipios y regiones, que ofrecian parcelas y edificios preexistentes complitendo con su localización estratégi$\mathrm{ca}^{35}$. El hecho de que la sede de Ferrocarriles Andaluces, una de las principales compañías del país, estuviera en

29. En la Conférence International de la Lumière en Lausanne y Leysin (1928) se trató la incorporación de espacios formativos y de trabajo.

30. Cfr. AZNAR, Adolfo. La vida de los ferroviarios y sus colegios de huérfanos. Documental. Madrid: 1944 [consulta: 20-02-2017]. Disponible en: https:// ww.youtube.com/watch?v=KrJIWcisis'

1. Hablando con nuestro arquitecto, op. cit. supra, nota 15 .

de este país, considerado como "el gran sanatorio de Europa". JIMÉNEZZ MORALES, Eduardo; VARGAS 政 2017 , vol. 24, $n^{\circ} 1$, . . . 248

33. Hablando con nuestro arquitecto, op. cit. supra, nota 15 .

34. El Sanatorio Antituberculoso en Zagreb (Croacia) STKZ, de Ennest Weissmann (1930) podemos considerarlo una "evolución tipológica del sanatotrio com pacto al de pabellones dentro de la morfologia en T'. RULLOBAA QUECEDO, op. cit. supra, nota 23, pp. 127-152.

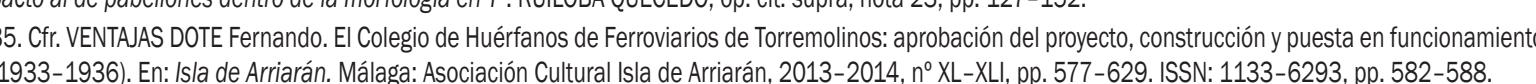
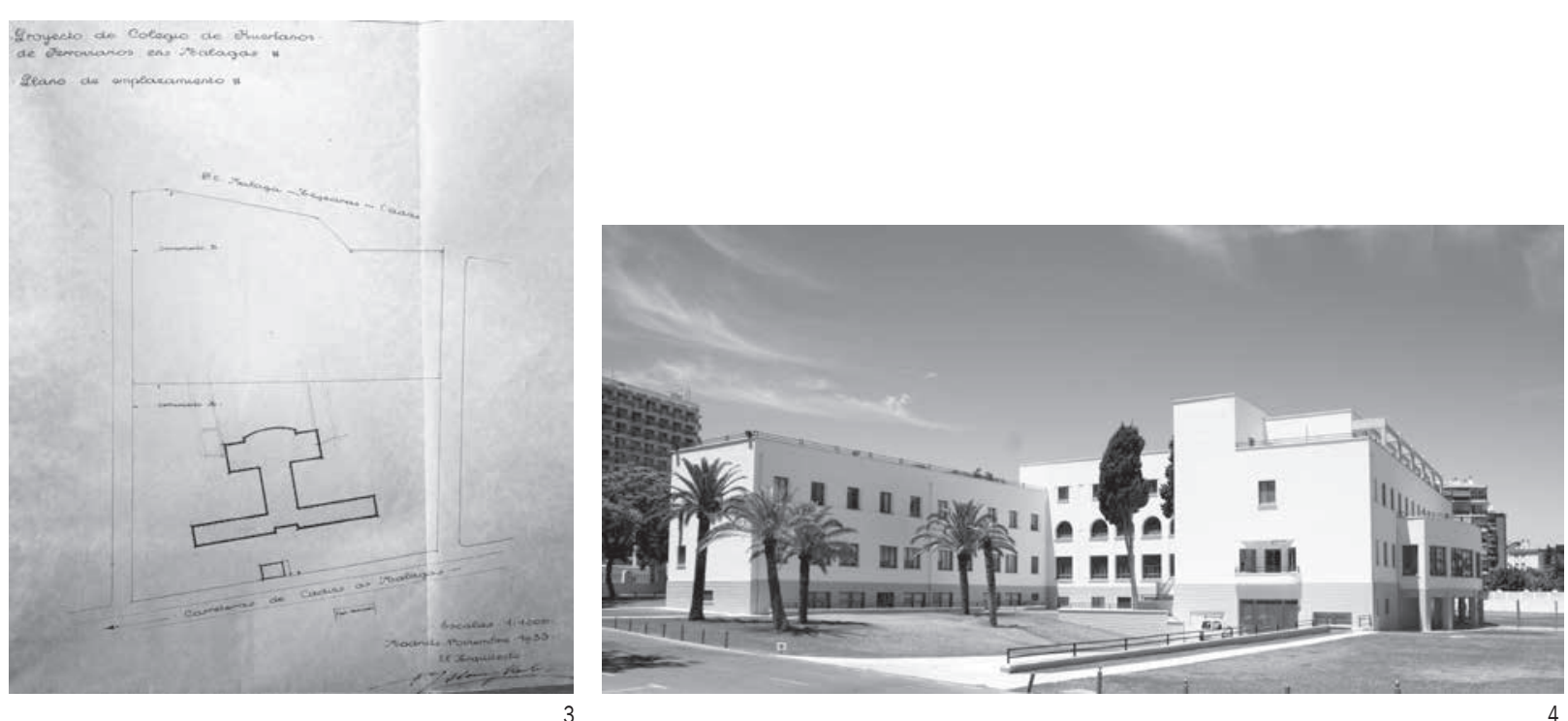

Málaga acabó decantando la decisión final. La parcela elegida tiene $22820 \mathrm{~m}^{2}$ y se localiza estratégican la costa y en allura sobre la tova de Toremolins, con el razado del ferrocaril en su limite este y la carretera Ḿ́ aga-Cádiz en el oeste (figura 3). El colegio tendra superficie construida de $2244 \mathrm{~m}^{2}$ con capacidad para 325 estudiantes, bastante menor a media de los distintos $0^{\circ}$ legios, que asciende a 450 estudiantes.

Alonso Martos busca clarificar y simplificar su propuesta madrileña, aspirando a enunciar la "interpretación perfecta de un colegio-orfelinatonse Se confirma en la elección del edíficio único, afirmando que la organización en pabellones aislados no está justificada ni siquiera en hospitales, cuestionando la eficacia de su desarrollo atomizad ${ }^{37}$, como ocurre con el Sanatorio Nacional de Torremolinos. Al mismo tiempo que desarrolla este proyecto, participa en concursos para colegios-internados para otras instituciones: prepararía una propuesta para el "Concurso de anteproyectos para un construcción para edificio destinado a hogar-escuela de huérfanos de Co rreos en la Ciudad Universitaria de Madrid" aunque no llega a presentarla por falta de tiempo $0^{38}$ Considera notables las propuestas ganadoras ${ }^{39}$ aunque estima que los $\mathrm{CHF}$ están más "adelantados": "El de Madrid, con seis años de

vida y con la rapidez que marchan ahora las cosas modernas, ni está anticuado ni lo estará en mucho tiempo, y en el de Málaga estimo que la solución es más eficaz, tanto en coste como en utilidad práctica"40.

Alonso Martos define su propuesta para Torremolinos como esquemática, una organización que es traslación casi directa del programa de usos: "la caracterítica principal de este edilifio es ser 'esquemático' en su traza. la representación construida del 'gráfico' de las necesidades de los huérfanos dentro del colegiont1 El proyecto aspira a la optimización del ́́rea construida evitando espacios inúties y eliminando elementos decortivos. Esto repercute en una eficacia económica siendo su coste mucho menor que en Madrid en relación a la capacidad. La solución "esquemátice" para Torremolinos la considera mejor que la opción madrileña que denominará "de orden más clásico"1"2. Además mejora el programa con una sal de usos múltiples: salón de actos, sala de fiestas, sala de estudio y cine.

Esta arquitectura, traslación legible de un programa se formaliza on geometrías simples de color blanco de cubiertas planas despojadas de toda compleijanco de mental de su antecesor (figura 4). Se desarrolla en dos piezas paralelas unidas transversalmente, clarificando su

6. El nuevo colegio, op. cit. supra, nota 17, p. 5.

37. Iden.

39. El programa era muy similar al de los CHF, aunque con una parcela insuficiente que no permitía aproyectar el edificio en tres o cuatro plantass. LAGARDE, Eduardo; AZPPURUA, Jose Manuel. Concurso de anteproyectos para la construcción de un eafificio destinado a hogar-escuela de huerfanos de Correos en Ciudad Universitaria de Madrid. En: Arquitectura, marzo-abril 1935, $\mathrm{n}^{\circ} 2$, pp. 58 - 64

40. El nuevo colegio, op. cit. supra, nota 17, p. 5 .

41. Idem.

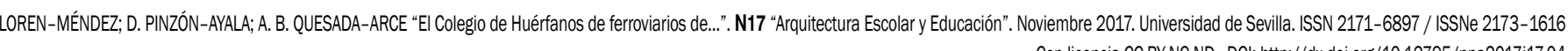



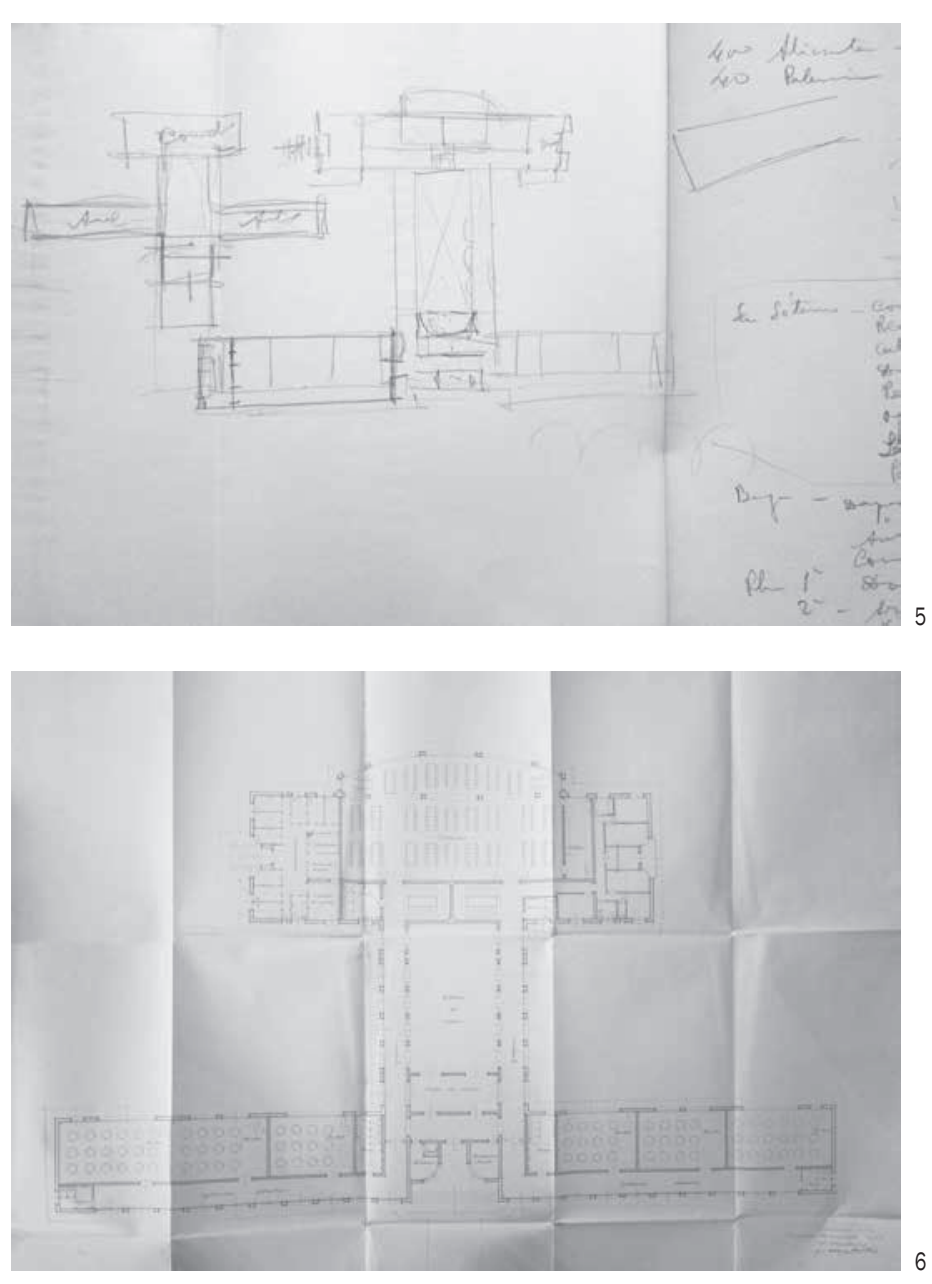

planta en doble T: el patio de Madrid queda aquí ocupado por el salón de usos múltiples. La colmatación del volumen refuerza su apuesta por la eficacia y simplicidad del edificio único, con geometrias que permiten la sectorización del programa hibrido docente residencial (figura 5). En la planta de acceso y organizando su fachada a la carretera, se sitúan las aulas con orientación Suroeste, con la entrada en el centro, reforzando el eje (figura 6). La galería porticada de las aulas como espacio dinámico ya referenciada, se formaliza con arcos de medio punto, elemento constante en sus proyectos, estableciendo en este caso un contraste con la simplicidad prismática (figura 7 ). Aparecen ya en Torremolinos los tres espacios para actividades complementarias. junto con el salón de usos multiples, el comedor y el recreo cubierto, los dos primeros localizados a eje en planta de acceso. Alojado en un volumen convexo con orientación Noreste, el comedor

es un mirador para los estudiantes, sivviendo como base de la metáfora de "un gran trasattántico encallado en la playa"na. Su volumen curvo da cobijo al recreo cubierto en planta semisótano, junto con los espacios servidores: cocinas, almacenes, espacios de lencería, duchas e insalaciones (figura 8)

El salón de usos multiples a doble altura conserva la ogica del patio: sus galerías porticadas, resueltas con los mismos arcos de medio punto que la galería exteior, consiryen una autentica fachada interior, al mismo tempo que resuelven la circulación entre las piezas an(tior y posterior (ifigura 9) (figura 10). La presencia de Lentes acentua esta vocación de patio reinterpretado. res, sirviendo las galerias en planta primera para separar el acceso de niños y niñas (figura 11). En planta segunda se localiza un dormitorio sobre el salón, dejando asi
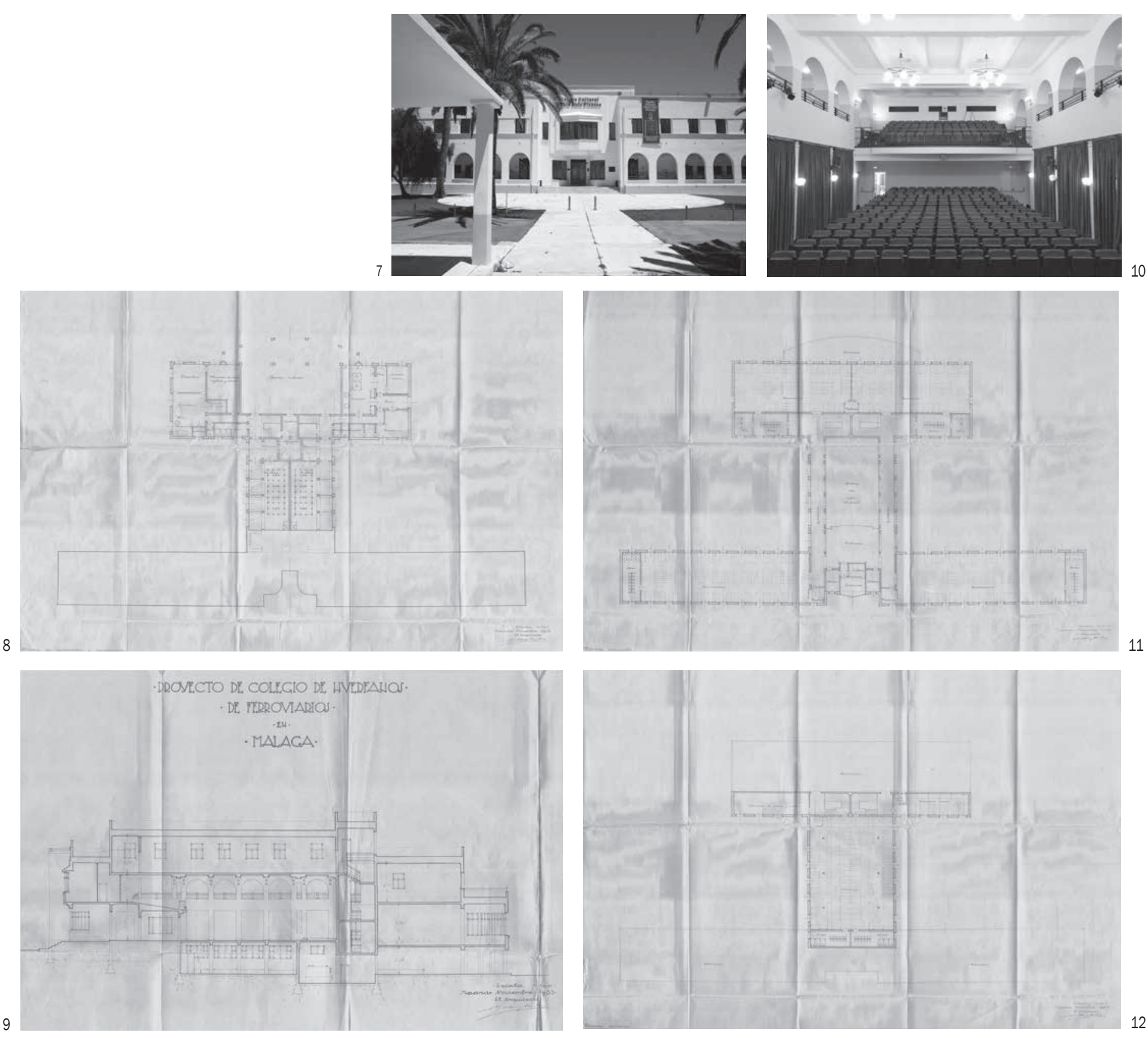

una gran terraza (figura 12). Dicha planta se amplía sobre 1958 para "aumentar su capacidad en otras 50 pla-

Variaciones arquitectónicas para el programa de CHF El hallazgo de los proyectos del resto de CHF nos desvela zas dose la parte centra de la fachada con dos terrazas en al menos complementando una primera hipotesis de un los extremos. Esta ampliación se barajó en 1944, con proceso de maduración, basada en la construcción de los proyecto de Alonso Martos que proponía, en contraste, mismos. Los ocho colegios restantes se proyectan entre construir en las esquinas de la pieza posterior, dejando 1943y 1945, comenzandose a construir entre 1945 y 1946. la terraza centrada; reincidiendo asi en el vacio del re-- El dificil momento de posguerra ralentizaria las obras, tercreo cubierto. minándose sólo cinco durante la década siguiente.

44. LOZANO AGÚNDEZ op cit. supra, nota 6 . p. 170 .

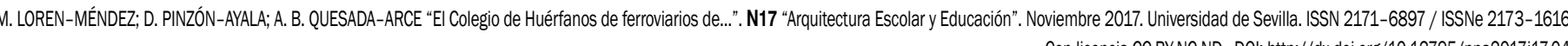


13. Variaciones. Croquis de volumetrías de Colegios Anso Martos, circa 1933ris. Atribuido a Francisco

14. Esquema de las variaciones tipolóficas de los
CHF de Torremolinos Vacarisses, Vilagarcía de Arousa, Alicante y Avilia, ordenados de las plantas inferiores a las superiores.

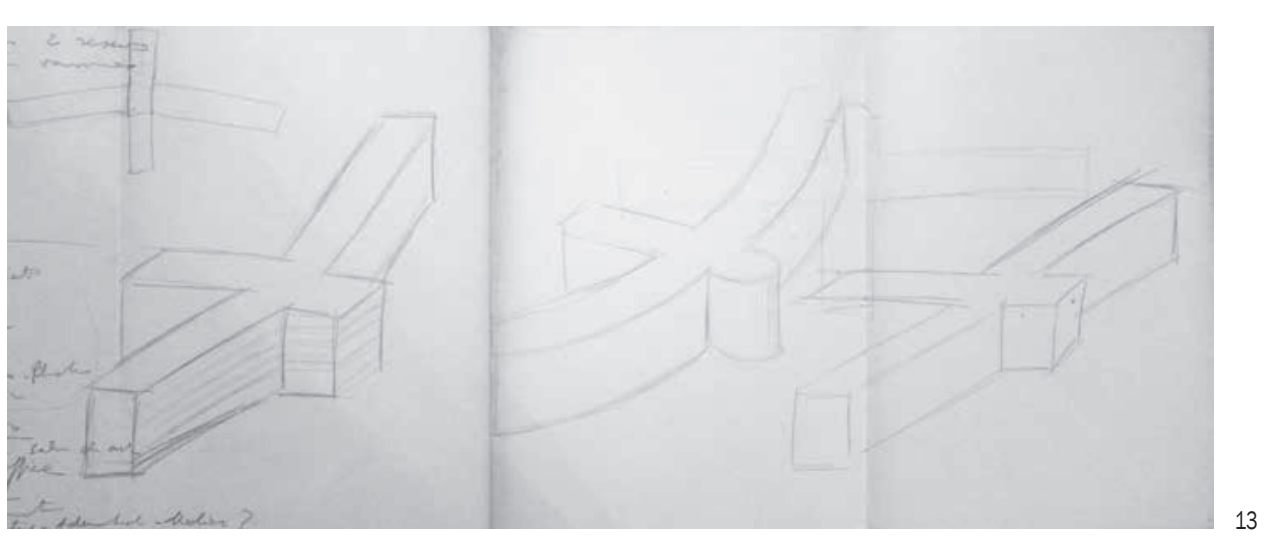

Se vislumbra una búsqueda desde el proyecto en la la pieza de fachada acoge tanto las aulas como el comeque muchos de los coleglos constituyen variaciones de dor, quedando el salón en la pieza perpendicular o "maruna propuesta programatica (figura 13). El estudio desve- tillo". Por ultimo, Avila se configura como una unica pieza, la en concreto un grupo de cuatro proyectos -Vacarisses, habiendose reducido al minimo el "martillo", que recogera Vilagarcia de Arousa, Alicante y Avila- que tienen al CHF la escalera y algunos espacios servidores. La galería porde Torremolinos como referencia (figura 14). Partiendo ticada en Alicante y Avila se entiende como extensión del ya del programa, el arquitecto continúa sus reflexiones programa del edificio, con la aparición de un pasillo posen torno a la sectorizacion, eficacia y orientación, proponiendo alternativas que atienden a la realidad de cada ubicación

Vacarisses reproduce la planta, orientación y sectorización de usos de Torremolinos en doble $\mathrm{T}$, con las aulas, comedor y salón en planta de acceso, recreo cubierto y espacios servidores en semisótano y dormitorios en planla superior. El recreo cubierto se situa bajo las aulas en la pastilla de fachada; acorta asi la distancia con la galería de las aulas, evitando el recorrido exterior entre ambos en un clima menos benigno que en Malaga. En Vilagarcía de Arousa las semejanzas en la traza son incluso mayores, incluyendo el volumen curvo en la pieza posterior de comedor sobre el recreo cubierto. La abrupta topografía del solar provocará sin embargo el escalonamiento de as piezas anterior y posterior, teniendo que desarrollar el programa en cinco plantas. Ubica en este caso las aulas en la fachada posterior; los dormitorios se localizan ahora junto al acceso, solución que no convence al arquitecto, aunque lo justifica por la esplendida vista de la Ría de Arosa ${ }^{445}$. Tanto en Vacarisses como en Vilagarcía de Arousa desaparece la galería porticada abierta, en adaptación
a las condiciones climáticas. En Alicante desaparece la pieza posterior, optando por una planta en T, en la que erior para independizar el acceso a las estancias.

En Vacarisses y Avila, la pastilla que resuelve la fachada con aulas en planta inferior $y$ dormitorios en la planta superior, se plantea de forma convexa -en la primera se abre en forma de lecha, en la segunda como una curvaque sigue la declinación solar. Este estudio no se orienta, en contraste con los sanatorios, a maximizar la exposición al sol, sino a gestionar la luzy el uso del espacio exterior, todo ello con la consideración de la zona climática. "perfectamente orientado con la declinación solar, con una distribución en curva que la sigue perfectamente-estudiada sobre bases cienlifias y exaclas la declinación medía del sol-, logrando con esto una iluminación y un soleamento perfectos ${ }^{146}$

De manera coetánea a estos colegios, el autor concibe y construye Palencia, León y Hondarribia, que representan búsquedas paralelas, con la aparición inevitable de la imposición simbólica del Régimen -que también está presente en Avila-. Los colegios en Castilla comparten así una formalización epidermica que los acerca a la arquitectura institucional de corte herreriano. En Paque , Alonso Marlos vielve a la estructura claustral a la pue superpone, sin mucho exilo, la traza en doble T. Una
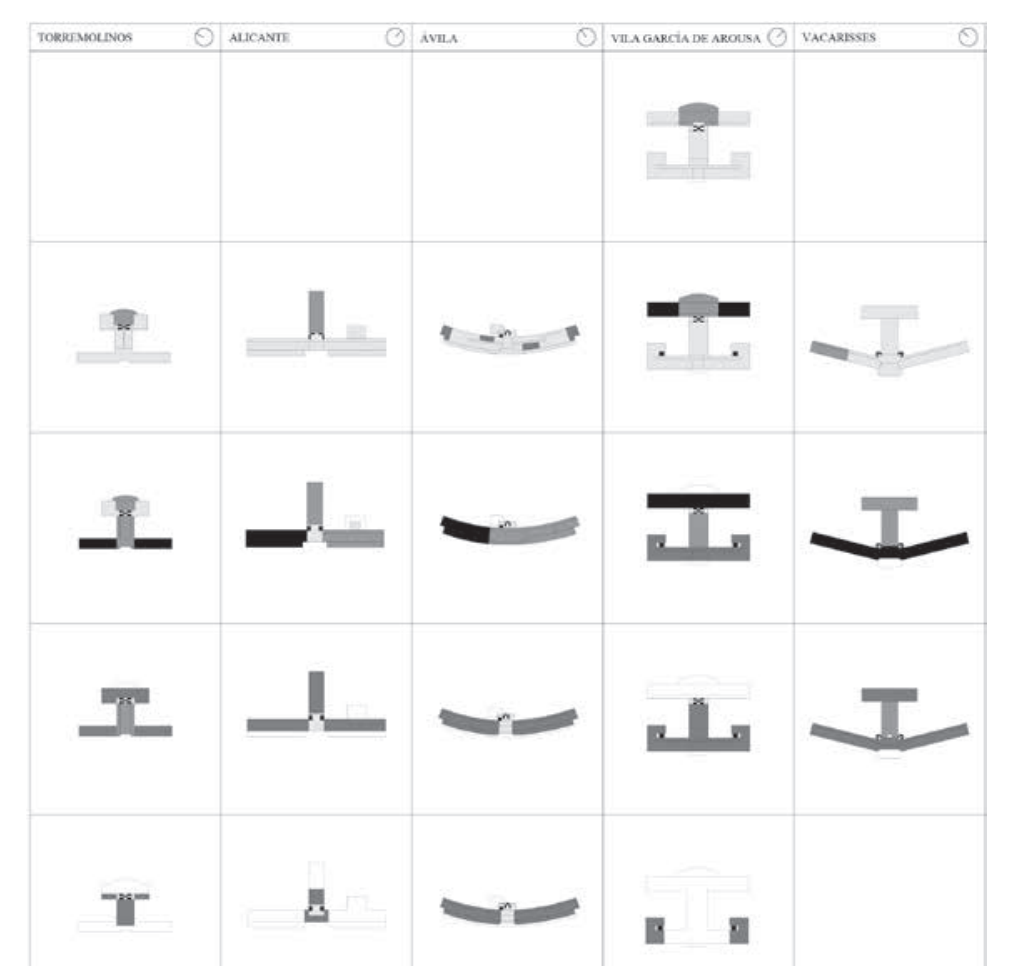

int

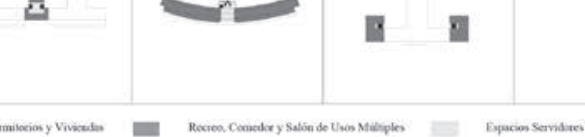

y complicando la claridad tipológica. En León se trabaja tambien con la idea de patio, esta vez màs cercano a las plazas de armas y no al espacio claustral. En las regiones más perifericas enfatizaria el uso de la tradición y la identidad regional: en Vilagarcía de Arousa utiliza las formas tradicionales de la arquitectura gallega; Vacarisses recuerda a una masia, la Casa Maternal de Dos Hermanas utiliza el regionalismo sevillano y en Alicante las referencias al Mediterraneo acercan a esta arquitectura al igual que en Torremolinos, a las arquilecturas de la Modernidad. En cualquier caso, todas las fachadas guardan claras semejanzas en su contiguración formal. la horizontalidad, la simetria y centralidad, asi como los arcos de medio punto, elemento que permanece incluso en aquellos casos donde desaparecen las galerías.

Modernidad de base téenica. Función y uso extensivo de hormigón armado

La escala de los CHF requiere una sistematización de su construcción. Sus usos exigen además una construcción

de grandes luces, para alcanzar el grado de diafanidad

necesario. El dominio del autor en el uso del hormigón hace evidente en obras como el Cine Salamanca en Madrid, obra publicada en Arquitectura y registrada por DoCoMoMo Ibérico. También utilizó el hormigón para las viviendas de ferroviarios, proyecto que supuso una re ferencia en un escenario que criticaba: "Bastaría exhibir fotografías de las últimas obras del Canadá, Budapest sur América, Charlotenburgo, y este Club [de fútbol de Zaragoza] de antes de la Guerra, para demostrar que andamos muy retrasados en obras valientes de Hormigón Armado"147. Esta cita nos pone en la pista de su conocimiento de obras con uso innovador y extensivo del hormigón armado48. Llega así a dominar el cálculo de estructuras, convirtiéndose en una referencia a nivel nacional e internacional. Los espacios definidos por potentes estructuras vistas de hormigón armado supusieron un factor decisivo, no sôlo en la eficiencia constructiva y la viabilidad económica, sino también en la experiencia interior que se desvela como componente diferenciador de su propuesta moderna. Ya empleado en Madrid, el uso extensivo del hormigón en todos los CHF Imprime a sus interiores un potente caracter. Esta honestidad estructura se refuerza con una estetetica derivada de la función, de una solución racional al programa habitacional y docente del alumnado.

En el CHF de Alicante indica la importancia de la durabilidad de los materiales para el uso docente y de internado: "sin más lujo que la buena calidad de sus materiales y su ejecución" entronca con una actitud tecnica y practica del espacio arquitectónico, con un compromiso con los nuevos materiales, con la economia de medios y con la durabilidad que debe regir la arquitectura educativa. Con independencia de los cambios operados en la aproximación formal externa, la búsqueda funciona programatica, asi como el uso honesto del hormigon armado, serían una constante en todos sus colegios. La Modemidad estaria siempre patente en sus interiores diáfanos enmarcados por estructuras desnudas de

77. Entrevista sobre el campo de fut-bol [sic]. Madrid: 20 julio 1956, inédito.

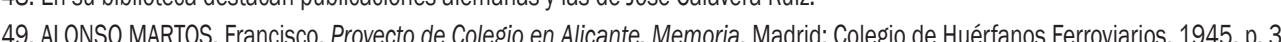




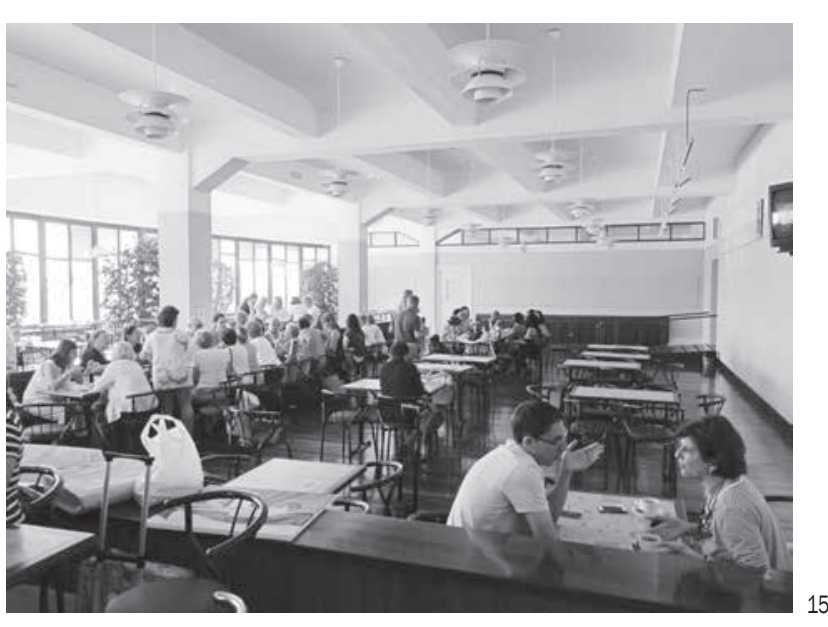

15. Colegio de Huérfanos de Ferroviarios de Torremolinos. Vista interior del comedor y la estructura
vista de hormigón, ahora cafetería tras la rehabilitación como Centro Cutural Pablo Ruiz Picasso. hormigón armado, en contraste con las concesiones estilísticas realizadas en cada momento" ${ }^{50}$ (figura 15).

EPILOGO. CIERRE, PROTECCIÓN Y RECICLAJE DOCENTE DEL CHF DE TORREMOLINOS

El CHF de Torremolinos cierra en 1973, habiéndose contemplado desde 1971 diferentes destinos para el edificio ${ }^{51}$ vinculados a su potencial turístico. Por el Rea Decreto 1341/1990, de 6 de noviembre, se declaró como BIC con categoria Monumento. En 1996 el Ayuntamiento convoca un concurso internacional para su rehabilitación como Centro Cultural. Su uso como Universidad Popular de Torremolinos asegura el compromiso mixto docente y social de la organización original cuestion clave para su recuperación ${ }^{52}$, complementado con la difusión de la cultura ${ }^{55}$. La desaparición del inernado, y las adaptaciones para su nuevo uso hacen

\section{Bibliografía citada:}

GGUIRRE Y PRADO, Federico. EI Colegio de Huérfanos Ferroviarios. En: Heraldo de Madrid, 1928, pp. 8.9

LLNSO MARTOS, Francisco. Proyecto de edificio para Colegio de Huérfanos de Ferroviarios en Málagáa. Madrid: Colegio de Huérfanos Ferroviarios, 1933 ALONSO MARTOS, Francisco. Proyecto de Colegio en Villagarcía de Arosa. Madrid: Colegio de Huérfanos Ferroviarios, 1945.

ALONSO MARTOS, Francisco. La Construcción de los Colegios de Huêrtanos de Ferroviarios (en leǵtítima defensa). Madrid: 1951.

ALONSO MARTOS, Francisco. La Construcción de los Colegios de Huérfanos de Ferroviarios (en leǵtíma defensa III). Madrid: 1953

50. "Talavera adoptóla modernidad en lo interior de sus obras [escuelas municipales] antes que en lo exterior o aparente". AÑón ABAAS, op. cit. supra, nota 12, p. 358 51. Cfr. LOZANO AGÚNDEZ, op. cit. supra, nota 6, pp. 306, 347,357 y 391

52. Entrevista con Victoria Acha (mayo 2016), gerente del Área de Urbanismo del Ayuntamiento de Torremolinos.

3. . Ff. Fus, Juan Pabo. Un

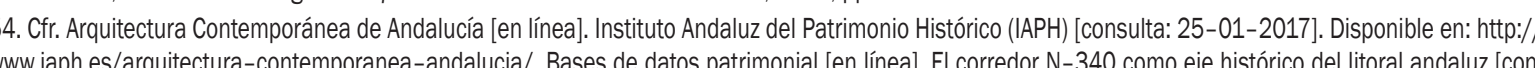

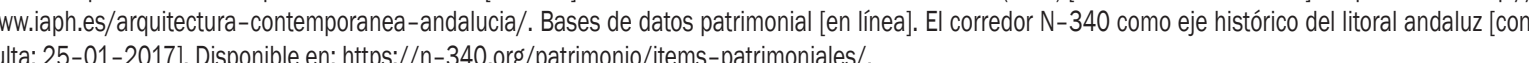
55. Cfr. PÉREZ ESCOLANo, Víctor et al. 50 años de arquitectura en Andalucía, 1936-1986. Sevilla: Junta de Andalucía, 1986, p. 248. W.A.A. Momo Andalucía Arquitectura del Movimiento Moderno en Andalucia, 1925-1965. Sevilia: Junta de Andalucía, 1999, pp. 56-59. LOREN-MÉNDEZ, Mar. La modedrnidad es

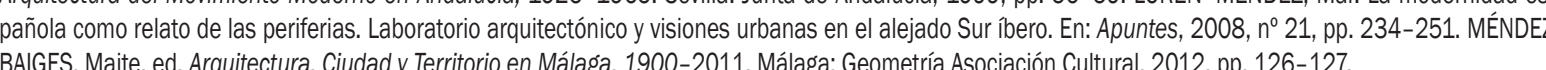

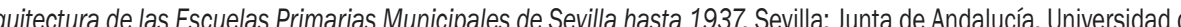
15, 2005.

AZNAR, Adolfo. La vida de los ferroviarios y sus colegios de huérfanos. Documental. Madrid: 1944 [consulta: 20-02-2017]. Disponible en: https:// AZNAR, Adolfo. La vida de los ferroviarios y
www.youtube.com/watch?v=KrJIWciSYYK.

CREMNITZER, Jean-Bernard. Architecture et Santé. Le temps du sanatorium en France et en Europe. Paris: Éditions A. et J. Picard, 2005. El nuevo colegio. En: Boletin oficial del Colegio de huérfanos de ferroviarios, agosto 1935, n 94, pp. 5-6.

Entrevista sobre el campo de fut-bol [sic]. Madrid: 20 julio 1956, inédito.

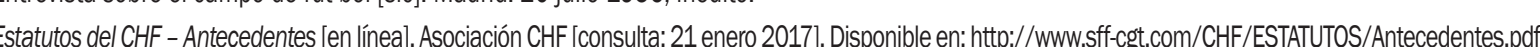
FUSI, Juan Pablo. Un siglo de España. La cultura. Madrid: Marcial Pons, 1999.

Hablando con nuestro arquitecto. En: Boletín Oficial del Colegio de Huếrfanos de Ferroviarios, $n^{\circ}$ extraordinario, 1930, p. 42

IMÉNEZ MORALES, Eduardo; VARGAS DíAZ, Ingrid Carolina. Hoteles y sanatorios: influencia de la tuberculosis en la arquitectura del turismo de ma

a Ascciación general de empleados y obreros de los Ferrocarriles de España yel problema de la vivienda. En: El Constructor, 1925, nº 20, pp. 494-497. LAGARDE, Eduardo; AIZPURÚA, José Manuel. Concurso de anteproyectos para la construcción de un edificio destinado a hogare-escuela de huérfanos

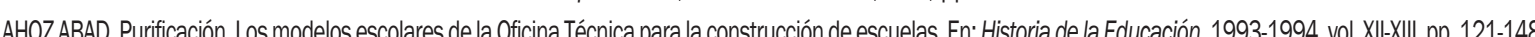
LÓPEZ MARTíN, Ramón. El magisterio primario en la dictadura de Primo de Rivera. Notas para su estudio. En: Historia de la educación: revista (3)

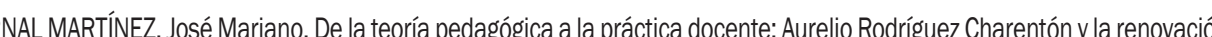
de las ciencias escolares en el primer tercio del sigilo XX. En: Homenaje al profesor A Afonono Capitán. Murcia: Universidad de Murcia, 2005, pp. 49-76. OREN-MÉNDEZ, Mar. La modernidad española como relato de las periferias. Laboratorio arquitectónico y visiones urbanas en el alejado Sur íbero. En: Apuntes, 2008, vol. 21, nº 2, pp. 234-251.

LOZANO AGÚNDEZ, Jesús. Evolución Histórico-Educativa Del Colegio de Huếrfanos de Ferroviarios (CHF). Una Propuesta Socio Laboral (1922-1996). D rectora: María Rosario Limón Mendizáabal. Tesis Doctoral. Universidad Complutense de Madrid, Departamento de Teoría e Historia de la Educación, 2016. MEENDEZ BAIGES, Maite, ed. Arquitecturara, Ciudad y Territorio en Málagá, 1900-2011. Málaga: Geometría Asociación Cultural, 2012. PEEREZ ESCOLANO, Víctor et al. 50 años de arquitectura en Andalucía, 1936-1986. Sevilla: Junta de Andalucía, 1986.

PRADOS LOPEZ, Manuel. Una mañana en el Colegio de Huérfanos de Ferroviarios de Torremolinos. En: Blanco y Negrro, 22 marzo 1936, pp. 74-79. Preventorio para huérfanos de ferroviarios, en Ávila. En: Cortijos y Rascacielos, 1952, nº 69, pp. 27-33.

RULLOBA QUECEDO, Cecilia. Arquitectura terapéutica. EI sanatorio antituberculoso pulmonar. Directora: Josefina González Cubero. Tesis Doctoral. Universidad de Valladolid, Departamento de Teoría de la Arquitectura y Proyectos Arquitectónicos, 2012.

RULOBA QUECEDO, Cecilia. Arquitectura sanitaria: sanatorios antituberculosos. Madrid: Escuela Nacional de Sanidad, Instituto de Salud Carlos III, Ministerio de Economía y Competitividad, 2014.

SANCHEZ COLANTES, Sergio. Ferrocarril e ideología en España a finales el XXX. Contribución a su estudio. En: IN Congresoso Historia Ferroviaria. Málaga: 2006. SANTOS, José. EI Asilo de Huérfanos Ferroviarios. Una idea plausible. En: FAdelantel... Revista Semanal Ilustrada de Ferrocarriles, $n^{\circ} 61,1912$, pp. 5 -6. VENTAAS DOTE, Fernando. EI Colegio de Huérfanos de Ferroviarios de Torremolinos: aprobación del proyecto, construcción y puesta en funcionamiento (1933-1936). En: Isla de Arriaran. Málaga: Asociación Cultural Isla de Arriarán, 2013-2014, n XL-XL, pp. 577-629. ISSN: 1133-6293.

W.AA. Momo Andalucía. Arauitectura del Movimiento Moderno en Andalucía, 1925-1965. Sevilla: Junta de Andalucía, 1999.

Mar Loren-Méndez (Malaga, 1968) Profésora Titular del Departamento de Historia, Teoría y Composición Arquitectónicas. Escuela Técnica Superior de Arquitectura. Universidad de Sevilla.

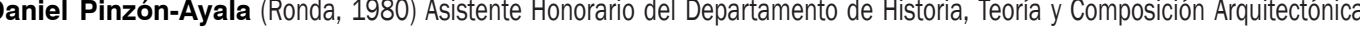
Escuela Técnica Superior de Arquitectura. Universidad de Sevilla. Ana B. Quesada-Arce (Benalmádena, 1972) Asistente Honoraria del Departamento de Historia, Teoría y Composición Arquitectó 


\section{EL COLEGIO DE HUÉRFANOS DE FERROVIARIOS DE TORREMOLINOS. UN EJEMPLO DE INNOVACIÓN DOCENTE}

THE TORREMOLINOS SCHOOL FOR ORPHANS OF RAILWAY WORKERS. AN EXAMPLE OF

EDUCATIONAL INNOVATION AS ARCHITECTURAL PROJECT

Mar Loren-Méndez; Daniel Pinzón-Ayala; Ana Belén Quesada-Arce

p.57 EDUCATIONAL DIMENSION AND SOCIAL MISSION: THE SPANISH RAILWAYS

The School for Orphans of Railway Workers and its chief architect: Francisco Alonso Martos

The Schoolfor Orphans of Railway Workers (SOR) (Colegio de Huérfanos Ferroviarios: CHF) is a charitable organization
founded in 1922 as a subsidiary of the Association of Employees and Workers of the Spanish (Asociación de Empleados y Obreros de los Ferrocarriles de España: AGEOFE), founded in 1888. Moves towards creating facilities for orphans of railway workers, originally denominated "asylums"', can be traced back to 1912 The educational dimension as a fundamental parameter of its social mission is defined by the Royal Order of the 12 May 1926 as providing a mixed proille or social and

In 1935 , it was specified that, in order to fulfil its mission, the association of SORs "shall build and sustain Schools
and other establishments for the education and instruction of orphans". The SOR association clearly aimed to build schools as one of its principle tasks so that "these would in themselves acquire such importance that their form would in time be mistaken for the aim"s'. The name chosen for the association is significant, emphasizing from the onset the

Research reveals that the architect contracted to carry out the SOR construction project was Francisco Alonso Martos ( (888, Granada -1961, Madrid). Along with the SORS, Alonso Martos created a network of shobols, some of
which include social centres in their programs. The architect also put forward proposals for garden cities for railway which include social centres in their programs. The architect also put forward proposals for garden cities for railway
workers - self-sufficient complexes that invariably incorporate building plans for a school, even if never built. He workers - self-sufficient complexes that invariably incorporate building plans for a school, even if never built. He
was appointed chief technician for charitable organizations belonging to the Spanish Railways', greatly contributing was appointed chief techniciaian for charitable organizations belonging to the Spanish Railways4, greatly contributing
to setting up a number of educational establishments, with a significant production in the definition of its various

p.58 by forming agricultural prison colonies recognized in a law passed in 1933 relating to vagabonds and miscreants carrying out projects on the Isla de Ons (Pontevedra), and in Oña (Burgos), as well as a similar project in Equatoria Guinea. He was also appointed architect by a number of public and private institutions including, among others, the Land Regl was do

Malaga), Alicante, Palencia, Leon, Avila, Vilagarcia de Arousa (Pontevedra). Dos and 1958: Madrid, Torremolinos (Gipuzkoa), and Vacarisses (Barcelona). Only the first two were created before, the Civil War, the Malaga complex being the only one whose planning and construction phases were completed during the Second Republic. As for the others,
the Vilagarcia de Arousa complex never succeeded in functioning as an SOR6 and the Hondaribia Vacarises Dos Hermanas complexes were never opened. The latter was designed as a maternity college, presenting a slightly different program (figure 1). During the dictatorship, the architect developed his work amid strong criticism, and his projects were poorly received. This would lead to his resignation as conservation architect of the existing Madrid and Torremolinos SORs, and, in 1953, as architect and director of works of those that were in the process of being built. Educational innovation and architecture

The SORS' educational proposal formalized "the values of progress and modernity"that were characteristic of associations linked to the railways in Spain (figure 2). Their vision was accommodated by reforms in the educationa system over the tirist tew decades of the twentitit century, with a particular impetus in the twenties, aiming to "remove educational reality"s. ideological instrumentation of education that would on occasion affect the SORs' educational project: at first, the SOA authorities appeared "undecided over secular or religious communities"s. Although lay education was finally adopted a chapel was set up in the Madrid SOR.

The Second Republic would be the political system most sympathetic to theSORs' educational project. Educational innovation was clearly a priority, giventhe appointment of Aurelio Rodifiguez Chanteron, regarded as a leading educationalis in Spain ", as dicational innovation was appointed director in Torremolinos: he would subsequently resign atter the militany yprising From 1922, the curriculum covered both primary and secondary education, the latter including preparation for high school qualifications - known as bachiller in Spain - and, by 1929, this included both the standard and the university bachiller. The planned educational program was intended for both boys and girls, with slight variations, in

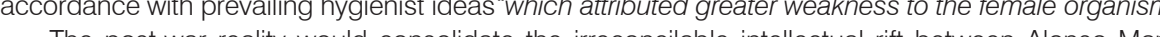

while religious authorties were put in charge of the different SORs. Despite this, there were no significant changes to Nevertheless, the SOR idea was conceived in a context of on-going debate relating to new concepts in education and teaching practice. In Spain, these were largely channelled "through the Free Institution of Education (Institución Libre de Enseñanzza) [.... also contributing to other institutions such as the National Pedagogical Museum (Museo Pedagogico Nacional)". These trendscombined with the hygienist proposals as a way to modernize and design Such functional aspirations led Alonso Martos to prioritize optimal lighting and ventilation, using "a modern system of aeration $n^{\prime \prime \prime}$, an architecture that looks outwards, and which establishes the distribution of space and the dimensions of the windows in relation to the orientation and the function of the rooms: "The project emerged by allocating a specific

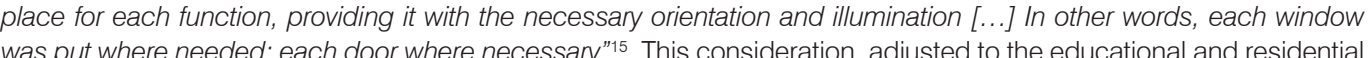
programs, contrasted, with healthcare architectures and their therapeutic use of the sun. In the case of the SOAf an optimal orientation was carefully considered from the hygienist point of view, integrated with the programmatic sectorial divisions and with the new trends in education where physical and creative activities in the open air are key. The SORs proposed two elements to ensure direct contact with the outdoors: firstly, the covered recreational area, which appeared in Torremolinos as an element of sufficient scale to ensure that students had easy access to the outdoors. The daily program emphasized outdoor games and creative activities as a fundamental part of
education: gardens were illuminated so that "the children could play at night"lis. In Torremolinos, the facility faces east towards the sea, presenting a beautiful view that was bound to play an active role in the developmentof children's capacities. Secondly, the porticoed galleries, situated to the west in Torremolinos, connected to the classrooms, and are considered by the architect to be "of great use and they provide the children with a good place to play with easy
access to the garden at recreation time ${ }^{\prime 17}$. The sectorial separation between these two zones succeeds in creating an

The covered recreation area and porticoed galleriesfigure in all the various SOR projects, with each emplacement education that highlighted the importance for children of play and contact with naturue. In 1935 , the School Director's functions widened to include the "physical and moral education of the students, with special attention to games, recreational breaks, outings, and distractions as extremely useful elements in modern didactic practice" 1 , providing an
open space to be freely explored, lose oneself in and discoverhidden corners. Terraces that connect to the dormitories are also elements that figure throughout the SOR network Terraces are a major feature of the school in Madrid, and subsequently, in Torremolinos, for example, their design becomes more sedate, respecting certain limitations of simplicity and efficiency, in order to keep within budget. In Torremolinos, the terraces are situated to the east, provide overhead shelter, once more take advantage of the sea views,and are clearly differentiated from classical therapeutic spaces. under the Franco regime and its vision of education. Aiming at self-sufficiency and close monitoring of tree tims meant that 'play' as a fundamental educational incentive at the SORs was substituted by training in farm-work,and an emphasis on sport, in line with the doctrines of the dictatorship. The covered porticoed galleries are configured as an extension of the program, reducing their former quality of freedom. In the Alicante School, the porticoed gallery, his time facing west, doubles up as a classroom and a summer dining hall. In Avvia, this programmatic strategy is appear as extensions to the dormitories -resembling the layout of healthcare galleries in sanatoriums. However the convex orientation -as opposed to concave- of the façade, seeks to achieve optimal illumination rather than maximum exposure to the sun.

As well as the sectorial divisions that providespaces for both educational and residential functions, thequestion of gender was also taken into account. The SORs were originally conceived as mixed sex schools, reinforcing a progressive vision of education. To maintain some separation, the stairwells are duplicated in the case of Torremolinos,
establishing symmetrical floors, copying the state school model for the segregation of sexes, allowing for dormitories for girls and boys on either side. Franco's dictatorship would impose the complete separation of the sexes, removing thus the need for a vertical sectorial division in schools. Torremolinos was assigned to girls in care between the ages of 8 and 12, after it was decided that girls should beneffit from enclaves with a gentler climate. The privileged spot on Wuring the summer season, the girls were moved to Alicante and Palencie "on the grounds of the rather unsuitable

ins


p.61 THE TORREMOLINOS SCHOOL FOR ORPHANS IN CONTEXT

The boarding schools created for orphans of railway workers thus all share a hybrid character, combining a function in social wellare with a strong opmintment to education. These are large-scale installations, transcending the strategic conditions as sun exposure, ventilation and the local area, including rail access - given its institutional link combined with road access. The buildings were planned to provide open air spaces covering an area larger than the building itself, according to standarddesignsfor the building of school and healthcare facilities²1. During the Franco

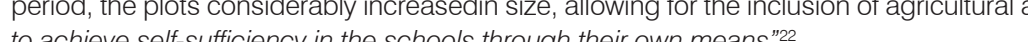

to the two architectural categories of healthcare pavilions or the Swiss-German model consisting of a single buildingez'. Such considerations relating to the atomization or the compacting of the program were framed within the prevailing hygienist theories and the need to separate and isolate specific uses. This debate was reflected at a national level with the development of both private and
public ant-tuberculosis hospitalse was favoured25 with one-off examples of the T-shaped model for the layout, resembling the form of an airplane, a typological development that would persist during the second republic and consolidate under Franco. After the "Compeltition for Proposals for 200, 300- and 400-bed Antituberculosis San contms" (1942), the winning solution proposed by Ernesto Ripollés, with a single floor arranged in a double T, "was established as a model'"26 Within the Plan At the same time, in view of the citis

Wical lack of school facilities, with existing buildings both precarious and insalubrious, educational architecture was also developing rapidly.School buildings gradually improved thanks to the the Ministry's concern"2z; the approval of new norms - the 1905, 1923 and 1934 technical hygiene instructions for school buildings - as well as the development of pedagogical and hygienist theories outlined above. Schools began progressively to
state schools The SORs established clear parallells between the two architectural categories, and, to a certain extent, contributed
to their debates, with the added particularity that SOR complexes had a dual purpose, not only serving as schools
thus thus increasing their programmatic complexity. This is reflected in the concern for sectorial divisions, especially With regard to the distributilon of residential spaces, and to ensuring contact whithe outdoors and its incorporation

A national network. Origins and overview of the SORs
The Madrid SOR, the first school to be planned and built,was conceived as part of a network with Madrid acting as the central entity of the system, and the other schools as branches ${ }^{30}$. While sites were being deliberated, Alsonso
Martos investigatedexisting referents in Spain, visiting boarding schools and sanatoriums. The architect concluded "the immense majority do not fuffit the necessary requirements"sil, with the exception of the Pernales (Bizkaia) boarding school; the Martime Sanatorium in Gorliz (Bizkaia) and the Martime Sanatorium on the Isla de Pedrosa (Cantabria). All are strategically implanted and propose a single building with a clear axial plan, a model that would be applied to

In Europe, Alonso Martos highlights boarding schools and educational facilities in Switzerland - Geneva, The latter is arranged into pavilions, indicating that Alonso Martos studied both typological lines. However he explains that there existed fewer institutions for orphans in northern Europe: lower birth rates and greater state provision in the event of the loss of a parent, with public facilities covering these demands, suggest a specificity to the problematic in he case of Spain

Sartos explicitly highlights the active participation of the association of SORs, considering the project to (I) adapted opinions of bothdirectorsandemployees: "I can therefore confirm that the School for Orphans building represents everyone's ideas, which I merely adapted"33.
The Madrid SOR initiated theresearch for the planning phase of future SOR complexesthat included: a typologica consideration of astand-alonebuilding for a hybrid boarding school program; concerns for the orientation and the climatic zone as the basic criteria for adapting to the location; inserting these architectures into the pedagogica debate,transcending the hygienist dimension; the extensive use of concrete. Regarding hygienist concerns, only
the heath centre was conceived as an isolated unit. The geometric definition characteristic of a double T layout emergedand was clarried in Torremolinos. In Madrid, the centrepiece is occupied by a patio ${ }^{34}$, the last bastion o the cloistered courtyard applied to the educational space, establishing an unresolved tension between the interio

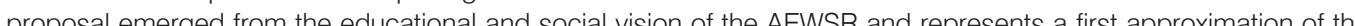

program, displaying certain excesses in decoration. The axial definition and the symmetry both characterize future

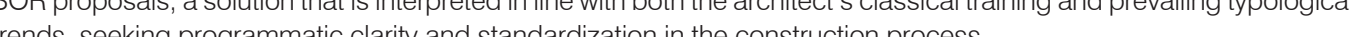
The Torremolinos SOR. The development of a referent

The Torremolinos SOR was the first branch of the network to be created. The facilities were planned by the relevant municipalities and regions, with offers of land or existing buildings competing on the basis of their strategic locations ${ }^{35}$. The fact that the headquarters of Andalucian Railways, one of the mainrailway companies in Spain, were situated in elevated geological formation in Torremolinos, and bordered by the railway lines on its easterly side and the Malagasubstantially less than the average of the other schools, which reached 450 students.

Alonso Martos tried to clarify and simplify his Madrid proposal, aspiring to express the "perfect interpretation of school-orphanage ${ }^{36}$. He validates his choice of a stand-alone buliding, asserting that a layout or isolited pavilions is not justrfiable even for hospitals, challenging thus the efficiency of suchatomized developments ${ }^{37}$, as applied to
the National Torremolinos Sanatorium. While this project was being developed, he participated in competitions for boarding schools for other institutions. he would develop a proposal for the "Competition for the preliminan plans for the construction of a building to seve as a home and school for orphans of the Post Office in the University City of

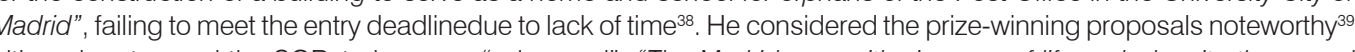
although esteemed the SORsto be more "advanced": "The Madrid one, with six years of life and, despite the speed

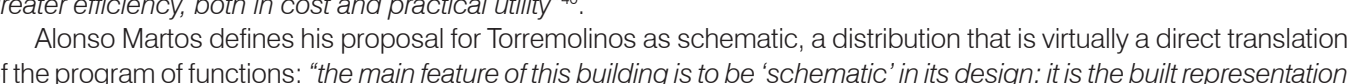
of a 'graphic' interpretation of the needs of the orphans at the school'pal. The project aspires to an optimal use of the

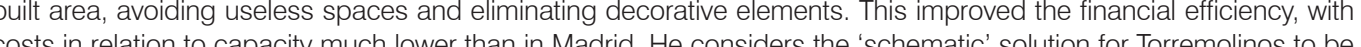
costs in relation to capacity much lower than in Madrid. He considers the 'schematic' solution for Torremolinos to be
an improvement on the Madrid option, which he describes as "more classical'tar. Moreover, the program is improved by a multi-purpose common room, senving as an assembly hall, study area, cinema or general venue.

The architecture, a legible translation of the program, is formalized into simple geometries that are white in colour with flat roofs and free of the decorative complexties of tits predecessor (figure 4). It is developed into two parallel units Inat are connected transversally, clarifying the double-T ground plan: in Madrid, the mutti-purpose common room
occupies the patio area. The compacted volume reinforces the aim for efficiency and simplicity of the stand-alone building. with geometries that allow sectorial divisions to accommodate the hybrid educational-residential program (figure 5). At access level, a central entrance reinforces the axis of the building whose main facade looks onto the road,
with the classrooms facing southeast (figure 6). Connecting classrooms and functioning as a dynamic space (as referenced above), the porticoed gallery is formalized by its round arches, a recurring element in his projects, in this activities now emerge: the multi-purpose common room, the dining room and the covered recreational area, the former wo situated at the axis at access level. Accommodated as a convex volume facing northeast, the dining room, like "a great deck chair stranded on the beach"nt3, serves as a mirador for students. The curved volume shelters the covered recreational area in the semi-basenent, as well as sonce tron

mmon room maintains the logic of the patio: its porticoed galleries, resolved of circulation between the front and back units (figure 9) (figure 10). The presence of fountains accentuates this choice of a reinterpreted patio.

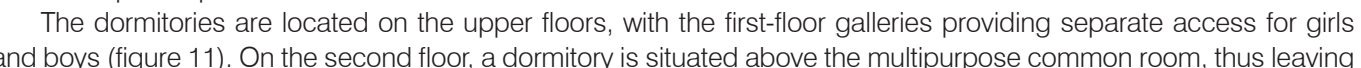
space to accommodate a big terrace (figure 12). The second floor was extended around 1958 to "increase capacity by another 50 places ${ }^{\text {m44, }}$, by adding to the façade and building two terraces, one at either end. This work was not carried out by Alonso Martos, atthoughanextension had previously been consideredin 1944, with a proposal by himto build on the conners of the façade, with its centred terrace positioned at the axis of the covered apeap

Architectural variations in the SOR program

Research into the remaining SOR projects reveals when they were first projected, undoing an initial hypothesis that a gradual design process had taken place. The eight remaining schools were projected between 1943 and 1945 , with
construction starting between 1945 and 1946. The difficult post-war years impeded construction, with only five SORS completed during the following decade. 
p.66 Findings suggest that many of the SORs constitute variations on a single programmatic proposal (figure 13). The stuady specrifically reveals that a group of four projects - Vacarisses, Vilagarcia de Arousa, Alciante and Avil -is based on the Torremolinos SOR as a reference (figure 14). Now using the program as a starting point, Alonso of each location

T. with the classrooms, dining room and multipurpose commonon room on the access floor the covered recreationa area and service areas in the semi-basement, and dormitories on the upper floor. The covered recreational area is situated below the façade unit, this serves to shorten the distance between the gallery and the classrooms, avoiding an exterior passage from one to the other in a climate that is less benign than Malaga's. In Vlagarcia de Arousa, the
design similarities are even more striking. including the curved volume of the front unit containing the dining room situated above the covered recreational area. The abrupt topography of the site would however lead to staggering the front and back units and the development of the program across five floors. In this case, the architect situates al the classrooms at the back of the bullaing, the dormitories are now situated next to the main front access, a solution

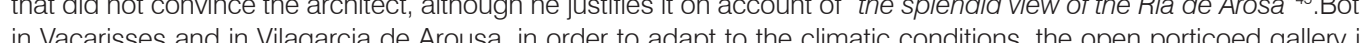
eliminated. In Alicante, the back unit disappears, opting for a T-shaped ground plan, where the façade shelters both the classrooms and the dining room, leaving the multipurpose common room to occupy the perpendicular unit or "hammer'.Finally, the school in Avila is configured as a single block, with the "hammer" reduced to a minimum and housing the stairwell and a number of serviceable areas. The porticoed gallery, present in both Alicante and Avila, is understood as an

In Vacarisses and Avila,the design that resolves the facade, with classrooms on the lower floor and dormitories
on the upper floor, proposes a convex form - in the former, it opens in the shape of an arrow, in the latter, as a curve - which is aligned to the sun's descent. Unlike the sanatoriums, this design is not oriented to maximize sun exposure,

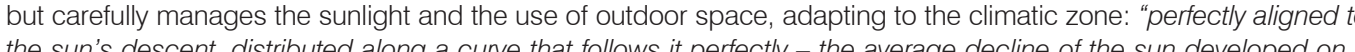
the sun's descent, distributed along a curve that follows it perfectly - the average decline of the sun developed on While building these schools, the architect simultaneously conceived then built the Palencia, Leon and Hondarribia SORs, representing parallel searches, with the inevitable emergence of the symbolic imposition of the new regime also present in Avila. The schools in Castille thus share a superficial formalization akin to the institutional architectures of Herrerian aspect. In Palencia, Alonso Martos returns to the cloistral structure on which he superimposes, withou
much success, the double T design. A central unit thus divides the patio, with walkways emerging that complicate the typological carty In Leon, the ide a fo a atio is once more addresed, on this occasion resembling a parade ground rather than a cloistral space. In the more peripheral regions, the use of tradition and regional identity are emphasized: in Vilagarcia de Arousa, he uses the traditional forms of Galician architecture; Vacarisses evokes a country house; the Casa Maternal de Dos Hermanas uses Sevillian regionalism; in Alicante, references to the Mediterranean infiltratte the architecture, and in Torremolinos, references to the architectures of Modernity are incorporated. Despite these
differences, all the facades retain clear similarities in their formal configuration. horizontality symmethy and centrality, as well as rounded arches, an element that is retained even in those schools where galteres are eliminated

Modern technical base. Function and extensive use of reinforced concrete

The scale of the SORsrequired a systemized approach to their construction. Moreover, their function required a large is reflected in buiddings such as the Cine Salamanca in Madrid, a work published in Arquitectura me usezine and listed in DoCoMoMo Ibérico. He also used concrete to build homes for railway workers, a project that became a reference in a scene that he criticized: "It would suffice to exhibit photographs of the latest constructions in Canada, Budapest, South America, Charlottenburg, and this pre-war [Zaragoza football] club, to demonstrate how behind we are in brav and extensive use of reinforced concretete $e^{48}$. He thus managed to master the structural mathematical rigor becoming reference at a national and international level. Spaces defined by powerful exposed structures of reinforced concrete represented a decisive factor, not only in the constructive efficiency and the financial viability of the project, but also regarding the inner experience, and this emerges as the differentiating component of his modern proposal. Already employed in Madrid, the extensive use of reinforced concrete throughout the SORs gives their interiorsa bold character

purposes: "with only the luxury of high quality materials and execution nay His modern discourse is associated with technical and practical approach to the architectural space, with a commitment to the use of modern materials, and the ap reonn concrete, would be constant features in all the schools. Modernity would always shine through his diaphanous p.68 interiors, framed by bare volumes of reinforced concrete, contrasting with the stylistic concessions that invariably occurred ${ }^{50}$ (figure 15)

EPILOGUE. CLOSURE, PROTECTION AND EDUCATIONAL RECYCLING OF THE TORREMOLINOS SOR From 1971 , alternatives related to the tourist potential of the Torremolinos SOR $\mathrm{R}^{51}$ were put forward and the orphanage cultural interest as a monument. In 1996, the Torremolinos Town Hall launched an international competition for the building as a cultural centre. Its current use as the Popular University of Torremolinos ensures the joint commitment to educational and social needs present in the original mandate, a key factor in its recuperation, complemented by essential to redesign the distribution and accessibility to the interior spaces

Since the end of the 1980s, the SORs have been listed in registers and databases of expert institutions in heritage gurchanship and protections, and on the basis of a tormal description, without specilying their typological contributions in the national or international a work of architecture in isolation, the SORs represent a complete series of works executed by a single architect in a short period of time, all key aspects in re-assessing their contribution in the hybridfield of educational and social welfare architectures: on all these counts, the Torremolinos SOR qualifies as a national reference.

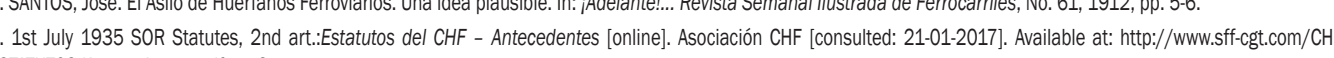
ESTATUTOSSAA

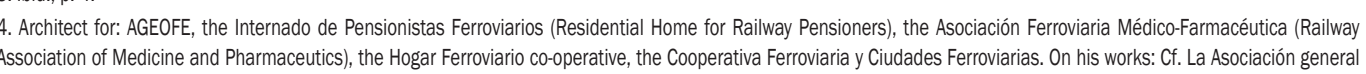

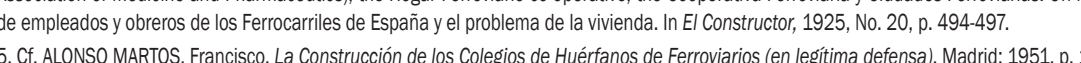

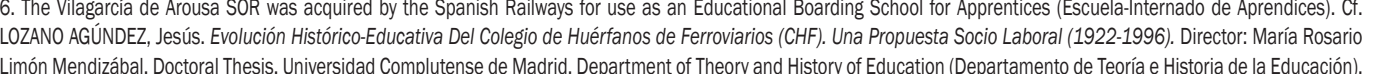
2016, . . 222.

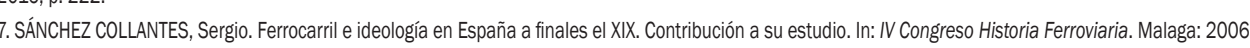
3. LÓPEZM ${ }_{5,0.0 .359}^{8.25}$

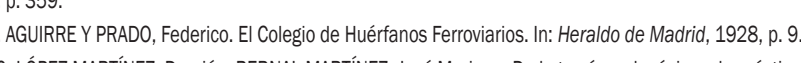

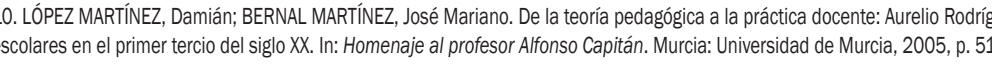

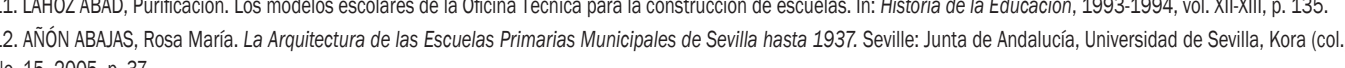

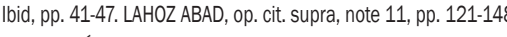

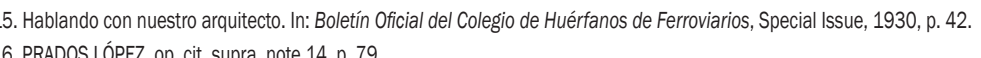

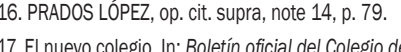

larios, August 1935, no. 94, p. 6.

19. I. bidi, p. .220.

20. Ibid, pp. 195-196.

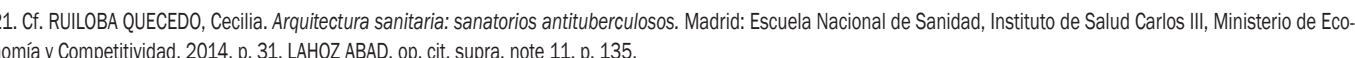

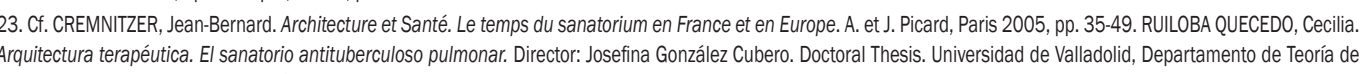

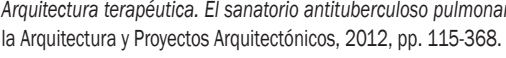

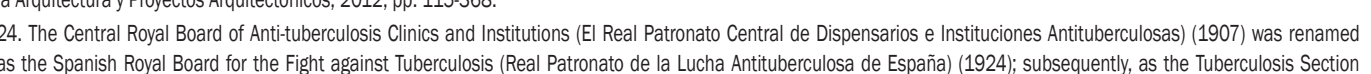

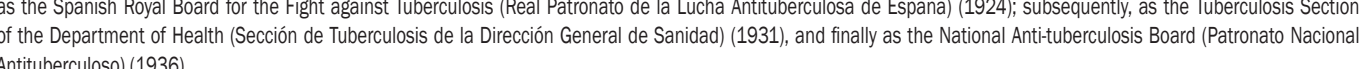

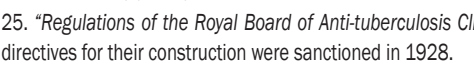

26. RULLOBA QUECEDD, op. cot. Supra, note 21, p. .66 
28. Ibid., p. 80 .

29. The incorporation of training and work spaces was discussed at the Conférence International de la Lumière in Lausanne and Leysin (1928).

30. Cf. AZNAR, Adolfo. La vida de los ferroviarios y sus colegios de huérfanos. Documentary. Madrid: 1944 [consulted: 20-02-2017]. Available at: https://www.youtube.com/ watch?v=KrJIWcfiSYk.

31. Hablando con nuestro arquitecto, op. cit. supra, note 15.

32. There is no mention that Alonso Martos visited healthcare architectures in Switzerland, a country that was considered to be "the great European sanatorium". JIMÉNEZ MORALES, Eduardo; VARGAS DÍAZ, Ingrid Carolina. Hoteles y sanatorios: influencia de la tuberculosis en la arquitectura del turismo de masas. In: História, Ciências y Saúde, January-March 2017, vol. 24, nº 1, p. 248.

33. Hablando con nuestro arquitecto, op. cit. supra, note 15.

34. The Anti-tuberculosis Sanatorium in Zagreb (Croatia) by Ernest Weissmann (1930) can be considered to be a "typological development from the compact sanatorium to a T-form layout of pavillions". RUILOBA QUECEDO, op. cit. supra, note 23, pp. 127-152. OK

35. Cf. VENTAJAS DOTE Fernando. El Colegio de Huérfanos de Ferroviarios de Torremolinos: aprobación del proyecto, construcción y puesta en funcionamiento (1933-1936). In: Isla de Arriarán. Málaga: Asociación Cultural Isla de Arriarán, 2013-2014, n XL-XLI, pp. 577-629. ISSN: 1133-6293, pp. 582-588.

36. El nuevo colegio, op. cit. supra, note 17, p. 5.

37. Idem.

38. Idem.

39. The program was very similar to those of the SORs, although the site was not adequate for "the projection of a three- or four-

floor building". LAGARDE, Eduardo; AIZPURÚA, José Manuel. Concurso de anteproyectos para la construcción de un edificio destinado a hogar-escuela de huérfanos de Correos en la Ciudad Universitaria de Madrid. In: Arquitectura, marzo-abril 1935, No. 2, pp. 58-64. OK

40. El nuevo colegio, op. cit. supra, note 17, p. 5.

41. Idem.

42. Idem.

43. PRADOS LÓPEZ, op. cit. supra, note 14, p. 76.

44. LOZANO AGÚNDEZ, op. cit. supra, note 6, p. 170.

45. ALONSO MARTOS, Francisco. Proyecto de Colegio en Villagarcía de Arosa. Madrid: Colegio de Huérfanos Ferroviarios, 1945.

46. Preventorio para huérfanos de ferroviarios, en Ávila. In: Cortijos y Rascacielos, 1952, No. 69, p. 28

47. Entrevista sobre el campo de fut-bol [sic]. Madrid: 20th July1956, unpublished.

48. German publications and works by José Calavera Ruiz can be highlighted in his library..

49. ALONSO MARTOS, Francisco. Proyecto de Colegio en Alicante. Memoria. Madrid: Colegio de Huérfanos Ferroviarios, 1945, p. 3.

50. "Talavera adopted modernity for the interiors of his works [local state schools] rather than for the exterior or the apparent". AÑÓN ABAJAS, op. cit. supra, note 12, p. 358.

51. Cfr. LOZANO AGÚNDEZ, op. cit. supra, note 6, pp. 306, 347, 357 and 391.

52. Interview with Victoria Acha (May 2016), manager of the Department of Urban Planning at Torremolinos Town Hall.

53. Cf. FUSI, Juan Pablo. Un siǵlo de España. La cultura. Madrid: Marcial Pons, 1999, pp. 74-75.

54. Cf. Arquitectura Contemporánea de Andalucia [online]. Instituto Andaluz del Patrimonio Histórico (IAPH) [consulted: 25-01-2017]. Available at: http://www.iaph.es/arquitectura-contemporanea-andalucia/. Heritage database [online]. El corredor N-340 como eje histórico del litoral andaluz [consulted: 25-01-2017]. Available at: https://n-340. org/patrimonio/items-patrimoniales/.

55. Cf. PÉREZ ESCOLANO, Víctor et al. 50 años de arquitectura en Andalucía, 1936-1986. Sevilla: Junta de Andalucía, 1986, p. 248. V.AA. Momo Andalucía. Arquitectura del Movimiento Moderno en Andalucía, 1925-1965. Sevilla: Junta de Andalucía, 1999, pp. 56-59. LOREN-MÉNDEZ, Mar. La modernidad española como relato de las periferias. Laboratorio arquitectónico y visiones urbanas en el alejado Sur íbero. In: Apuntes, 2008, n 21, pp. 234-251. MÉNDEZ BAIGES, Maite, ed. Arquitectura, Ciudad y Territorio en Málaga, 1900-2011. Malaga: Geometría Asociación Cultural, 2012, pp. 126-127. 


\section{Autor imagen y fuente bibliográfica de procedencia}

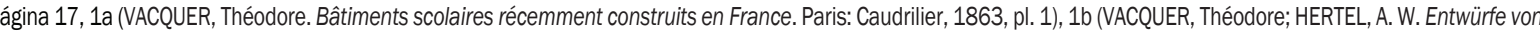

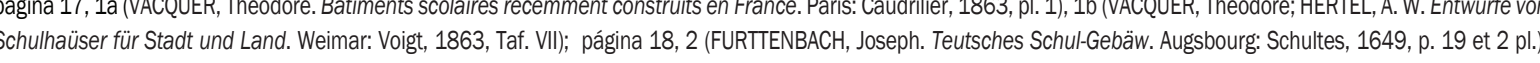

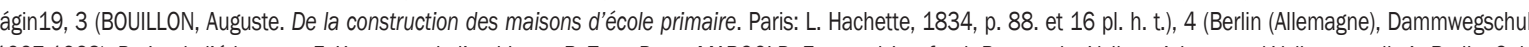

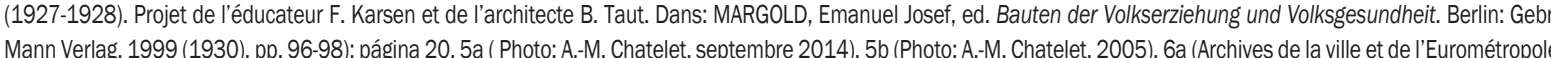
(1) Maquette de la grande percéé; E. Maechling, Musée historique de Strasbourg. Ophoto Musées de Strasbourg, M. Bertola), 7b (GOURLER, BIET, GRILLON et Feu TARDIEU.

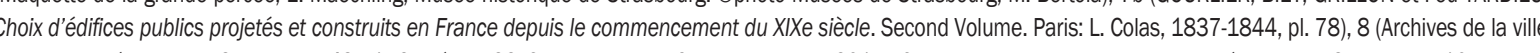

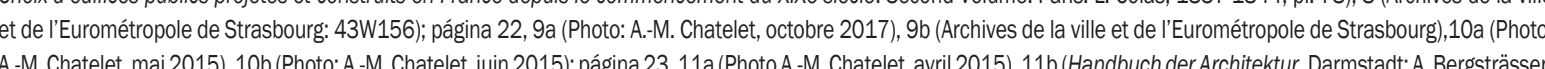

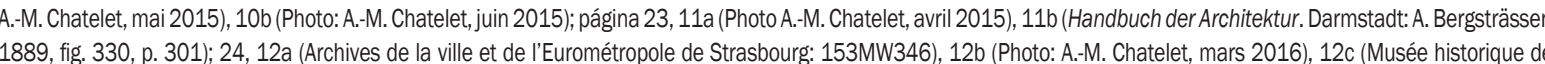

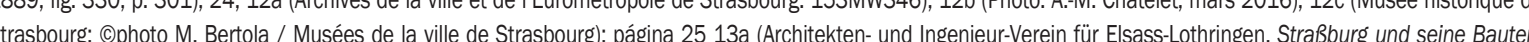
Strassburg: K. J. T. Trübner, 1894, p. 312), 136 (Photo: A.M. Chatelet, décembre 2013), 14a (Photo: A-M. Chatelete, avili 2016), 14 b (Photo: A.M. Chatelet, mai 2015); página

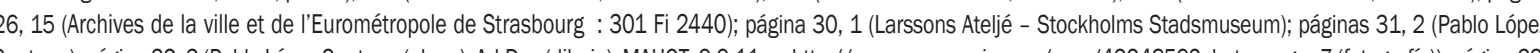

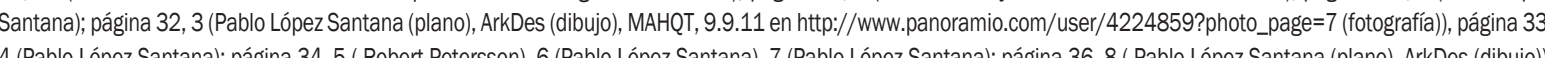

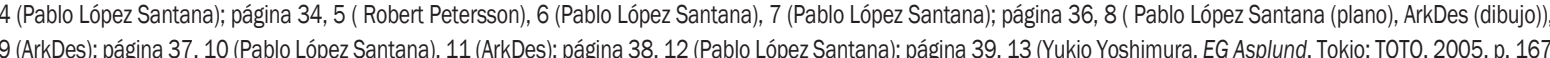

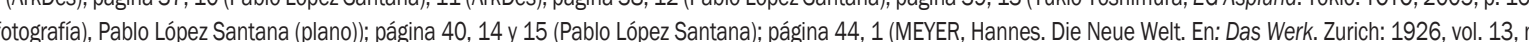
7, p. 218), 2 y 3 (KIEREN, Martin. Hannes Meyer. Dokumente zur Frühzeit Architektur - und Gestatungsversuche 1919 - 1927. Heiden: Niggl, 1990, p. 18; página 47, 4

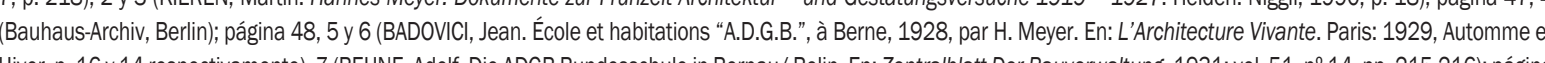

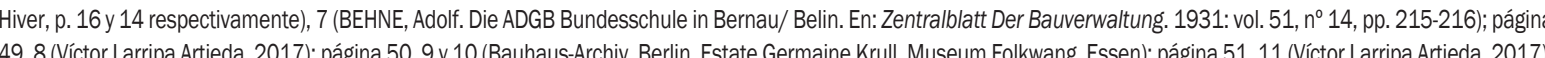

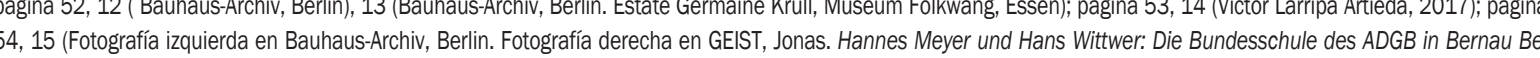
Berlin: 1930-1983. Potsdam: Potsdamer Verlags Buchnandlung, 1993, p. 16); página 59, 1 (Elaboración propia a partir de difierentes fuentes. Dibujo realizado por Roberto

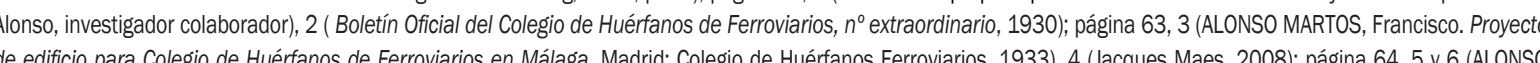

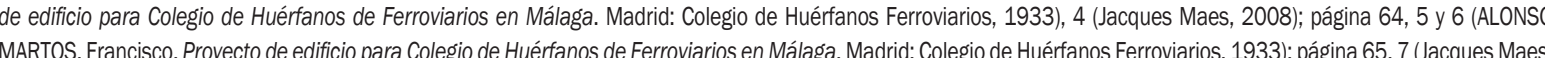

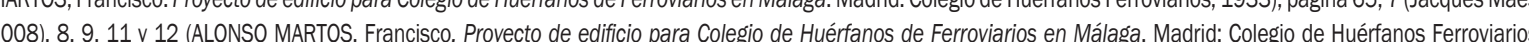
1933), 10 (Mar Loren-Méndez, 2016), 11 (ALLONSO MARTOS, Francisco. Proyecto de edificio para Colegio de Huếranos de Ferroviarios en Málaga. Madrid: Colegio de

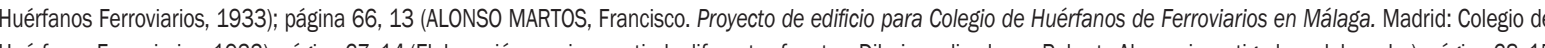

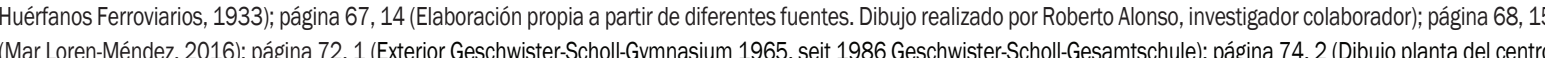

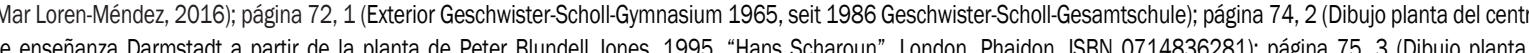
xonometría de aula para el grupo inferior, grupo intermedio y el grupo superior para el provecto del colegio en Darmstadt a partir de hitp hlescolano.blosspotcon

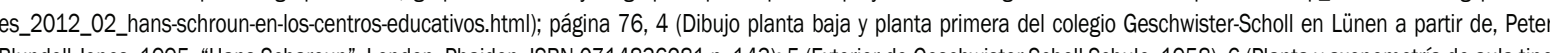
Blundell Jones, 1995, "Hans Scharoun", London, Pharidon. ISBN 0714836281.p. 143); 5 (Exterior de Geschwister-Scholl.Schule, 1958), G 6 Plantay axonometría de aula tipo en el colegio Geschwister-Scholl en Lünen a partir de, Peter Blundell Jones, 1995, "Hans Scharoun", London, Phaidon. ISBN 0714836281 p. 142), 7 Vista interior aula

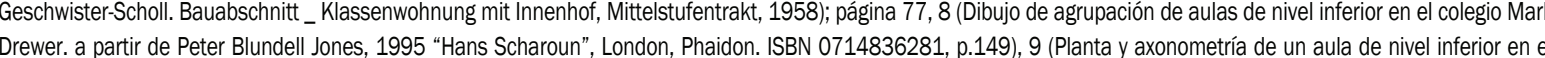
colegio Marl-Drewer. a partir de Peter Blundell Jones, 1995 "Hans Scharoun", London, Phaidon. ISBN 0714836281, p.149); página 78, 10 (Teatro del colegio Geschwister.

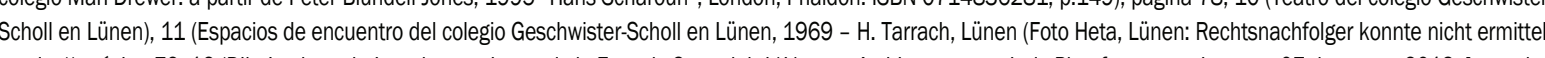

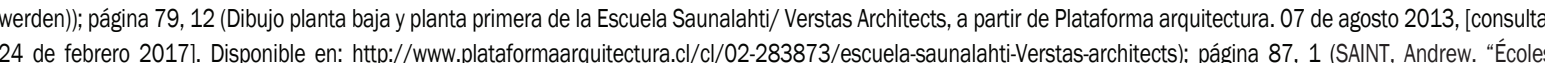

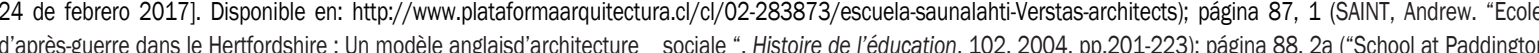

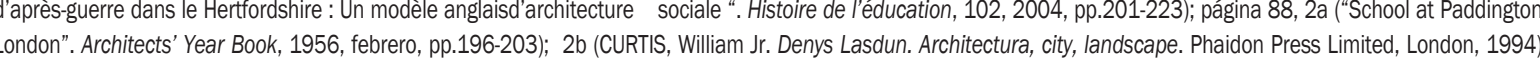

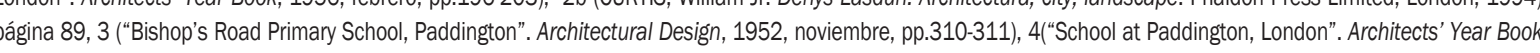
1956, febrero, pp.196-203), 5a "'School at Paddington, London". Architects's Year Book. 1956, feberero, p.196-203), 5b ""Bishop's Road Primary School, Paddington"

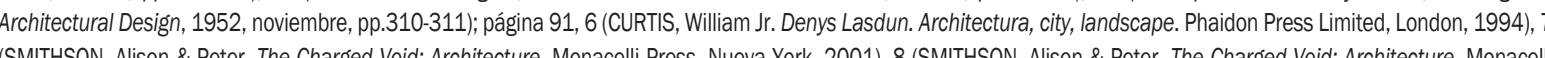

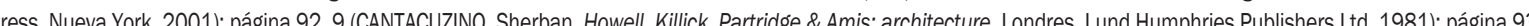

10 (CANTACUZINO, Sherban. Howell, Killick, Partridge \& Amis: architecture. Londres, Lund Humphries Publishers Ltd, 1981), 11 "PPimlico Comprehensive". Architectura

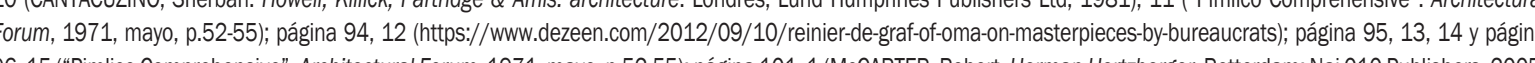
96, 15 ("Pimlico Comprenensive". Architectural Forum, 1971, mayo, p.52-55); página 101, 1 (McCARTER, Robert. Herman Hertzerger. Rotterdam: Nai 10 Publishers, 2005 ,

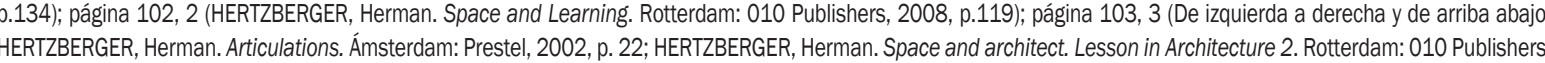

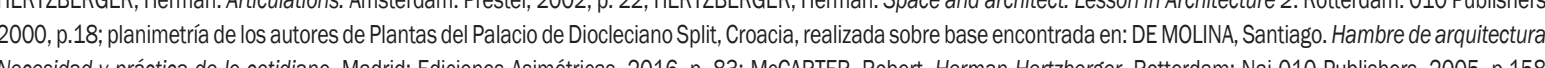
政

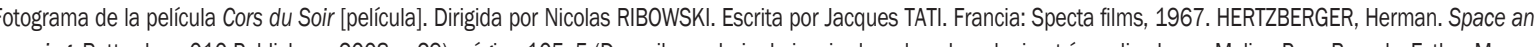

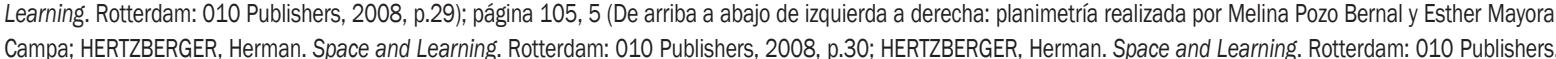

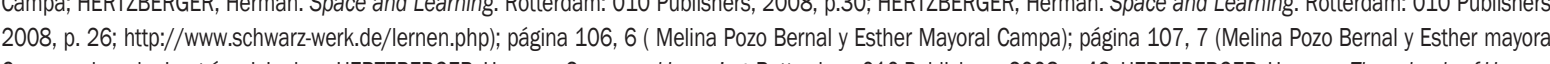

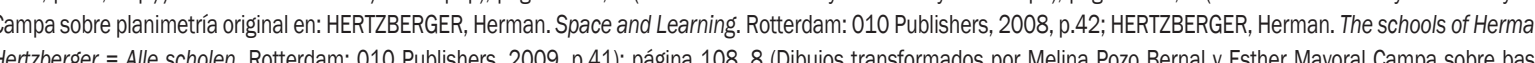
original en: HERTZZERGER, Herman. Space and Learming. Rotterdam: 010 Publishers, 2008, p. 83);página 109, 9 (Melina Pozo Bernal y Esther Mayoral Campa sobre planimetría existente en: MCCARTER, Robert. Herman Hertzberger. Rotterdam: Nai 1010 Publishers, 2005, p. 144 Y HERTZZERGGER, Herman. The schools of Herman Hertzberger

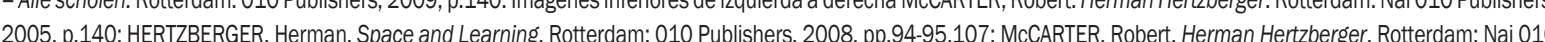

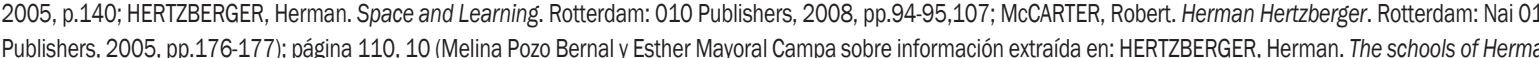
Hertzberger = Alle scholen. Rotterdam: 010 Publishers, 2009, pp.130,118. Fotograffias de arriba a abajo McCARTER, Robert. Herman Hertzberger. Rotterdam: Nai 010 Publishers, 2005, pp.184, 188, 185); página 111, 11 (Melina Pozo Bernaly Esther Mayoral Campa sobre información extraída en: McCARTER, Robert. Herman Hertzberger. Roterdam. Warto

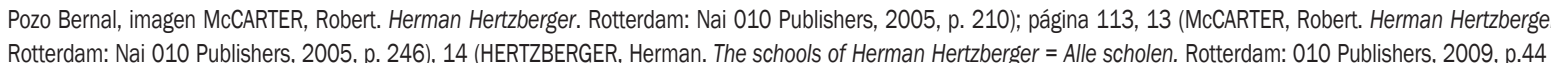
Mextraída en:

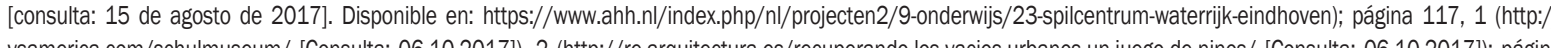

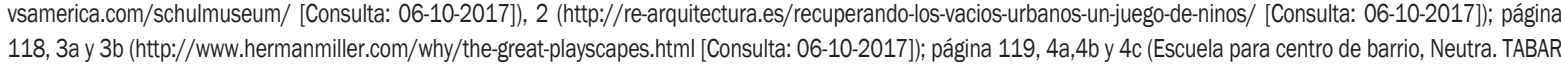

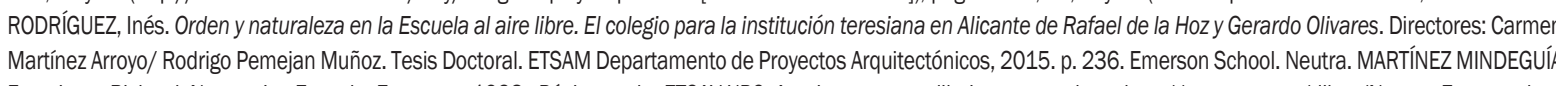

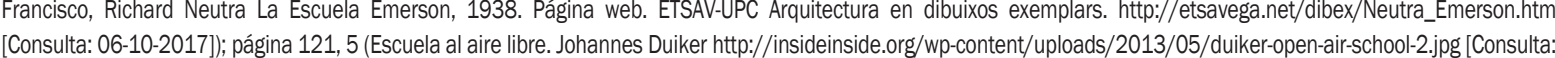

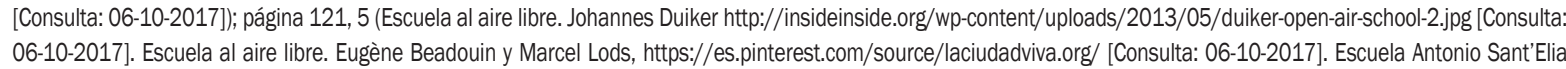

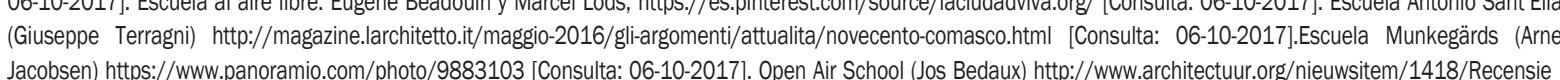

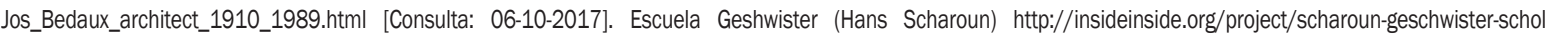

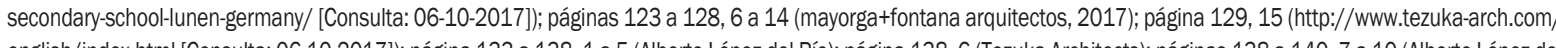

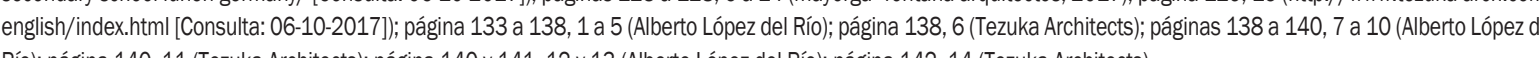

